KYUSHU-HET-110

TU-808

OU-HET 595/2008

\title{
Multi-Higgs Mass Spectrum in Gauge-Higgs Unification
}

\author{
Kentaro Kojima ${ }^{a} *$, Kazunori Takenaga $a_{+}$, and Toshifumi Yamashita ${ }^{c}: t$ \\ a Department of Physics, Kyushu University, Fukuoka 812-8581, Japan \\ ${ }^{b}$ Department of Physics, Tohoku University, Sendai 980-8578, Japan \\ ${ }^{c}$ Department of Physics, Osaka University, Toyonaka, Osaka 560-0043, Japan
}

(January, 2008)

\begin{abstract}
We study an $S U(2)$ supersymmetric gauge model in a framework of gauge-Higgs unification. Multi-Higgs spectrum appears in the model at low energy. We develop a useful perturbative approximation scheme for evaluating effective potential to study the multiHiggs mass spectrum. We find that both tree-massless and massive Higgs scalars obtain mass corrections of similar size from finite parts of the loop effects. The corrections modify multi-Higgs mass spectrum, and hence, the loop effects are significant in view of future verifications of the gauge-Higgs unification scenario in high-energy experiments.
\end{abstract}

\footnotetext{
*E-mail: kojima@higgs.phys.kyushu-u.ac.jp

$\dagger$ E-mail: takenaga@tuhep.phys.tohoku.ac.jp

${ }^{\ddagger}$ E-mail: yamasita@het.phys.osaka-u.ac.jp
} 


\section{Introduction}

The standard model (SM) of particle physics successfully describes high-energy experimental data. However, the SM involves the theoretical difficulty referred to as "hierarchy problem": due to large quantum corrections, the energy scale of the Higgs potential tends to become similar to the cutoff scale of the SM, in spite of the fact that the energy scale should be similar to the known electroweak one. This suggests that physics beyond the SM appears near the electroweak scale.

Among several candidates of the physics beyond the SM, unification of gauge and Higgs fields, so-called gauge-Higgs unification, is known as a promising idea with the help of compactified extra dimensions [1, 2, 3]. In the gauge-Higgs unification scenario, some of the extradimensional components of gauge fields are identified to Higgs fields at a low-energy regime. Phenomenologically viable electroweak Higgs sectors are known to appear with orbifold compactification of the extra dimensions. An advantageous point of the idea is finiteness of the Higgs potential even at some loop levels; hence, the potential is not sensitive to unknown ultra-violet (UV) physics, and the scenario gives a reasonable resolution to the hierarchy problem. The gauge-Higgs unification scenario has been extensively studied [4, 5, 6, 7]. In addition to the case with flat compactified extra dimensions, the case with a warped extra dimension has been also investigated [8]. The Higgs sector is predictive thanks to the higher dimensional gauge invariance. Namely, the scenario predicts light Higgs scalars, which can be a key ingredient for the experimental test of the scenario at LHC and ILC.

One of the most important subjects is to minutely examine the Higgs mass spectrum in the gauge-Higgs unification. Several models have been proposed and the mass spectrum has been studied. An interesting observation is that multi-Higgs scalars appear in some models. The models lead to non-vanishing tree-level Higgs potential which has flat directions; it has revealed that finite radiative corrections to the flat direction lead to the correct electroweak symmetry breaking dynamically [9, 10]. The Higgs scalars associated with the flat directions are massless at the tree-level and become massive through the radiative corrections; their masses have been studied by the one-loop effective potential [11, 12], or even at the two-

loop level in a simple model [13. The other modes among the multi-Higgs scalars, which are associated with the non-flat directions, are massive at the tree-level. Loop corrections to their masses, however, have not been focused on even though they are crucial to verify the Higgs mass spectrum in the models. It is also important to examine the spectrum in detail for the experimental test of the scenario.

In addition, the loop corrections to the non-flat directions are considered to be significant 
for determining the vacuum structure of the multi-Higgs potential. If the corrections are taken into account, then vacua deviated from the flat directions of the tree-level potential may appear. Clearly, such a vacuum structure cannot be studied when one focus only on the loop corrections to the flat directions. Thus, to reveal the correct vacuum structure of multi-Higgs models, the loop corrections to the non-flat directions should be incorporated.

In this paper, we study the multi-Higgs mass spectrum based on one-loop effective potential in a simple 5D model. We take bulk loop corrections into account for all the modes of the Higgs scalars: we study not only the loop corrections for the Higgs scalars along the flat direction of the tree-level potential, but also the ones for the Higgs scalars along the non-flat direction. As mentioned above, in the past studies, the latter has not been focused on though it is important. In our analyses, it is found that the one-loop corrections involve an UV divergence, which is proportional to the tree-level potential and is renormalized. The one-loop corrected multi-Higgs mass spectrum is derived through the effective potential after eliminating the divergence. It turns out that both the tree-massless and massive Higgs scalars have mass corrections of similar size from finite parts of the one-loop effects. Consequently, the loop effects modify multi-Higgs mass spectrum and are significant in view of verifications of the scenario in high-energy experiments.

The outline of the paper is as follows. In Section 2, an overview of multi-Higgs mass spectrum in the gauge-Higgs unification scenario is presented. In Section 3, we examine the one-loop corrected multi-Higgs mass spectrum in a simple 5D model. A perturbative calculation of the effective potential is developed to estimate the loop corrections. Summary and future perspective are given in Section 4. In Appendix A, field dependent operators that are needed to evaluate the effective potential in the model are presented, and Appendix B provides the evaluation of loop momentum integrals with the summation of Kaluza-Klein $(\mathrm{K}-\mathrm{K})$ modes.

\section{An overview of multi-Higgs mass spectrum}

In this section, an overview of the multi-Higgs mass spectrum is given. An interesting observation is that multi-Higgs scalars are predicted in some models of the gauge-Higgs unification. Models with two extra dimensions compactified on the orbifold $T^{2} / Z_{2}$ are illustrative examples; at a low-energy regime, the two extra dimensional components of a 6D gauge field behave as a pair of scalar fields, those are identified as two Higgs scalars [9]. It is also known that there appear multi-Higgs scalars in the 5D models with supersymmetry (SUSY) [10, 14]. Due 
to the SUSY, a real scalar field is accompanied by a extra dimensional component of 5D gauge field (and a Weyl fermion) to form $4 \mathrm{D} \mathcal{N}=1$ chiral superfield [15]; two Higgs scalars hence appear at low energy.

In the above models, the low-energy effective theory has a tree-level Higgs potential, where flat directions appear. Among the Higgs scalars, a mode, which corresponds to a flat direction and is a part of the zero modes of the extra dimensional component of the gauge field, triggers off the electroweak symmetry breaking. Quantum corrections to the flat direction determine the physical vacuum of the theory in terms of the non-zero vacuum expectation value (VEV) of the Wilson line phase degrees of freedom. Such the dynamical gauge symmetry breaking is known as the Hosotani mechanism [3].

We refer to the Higgs scalar that is the relevant mode to the symmetry breaking as the Symmetry-Breaking (SB) Higgs hereafter. The SB Higgs is identified as a zero-mode of extra dimensional components of gauge fields. In addition, gauge fields, non-SB Higgs scalars and K-K modes generally appear. The one-loop effective potential that takes account of only the SB Higgs background has been studied; the correct dynamical electroweak symmetry breaking has been shown to occur through the VEV of the SB Higgs [9, 10].

After the symmetry breaking, there appear the massive gauge fields, whose mass scale $M_{W}$ should be much smaller than the typical mass scale of the $\mathrm{K}-\mathrm{K}$ modes, $\mathcal{O}(1 / R)$, where $R$ is the radius of a compactified dimension. In the $5 \mathrm{D}$ case, for instance, the mass of the gauge boson is typically given by $\alpha / R$, where $\alpha$ is the VEV of the Wilson line phase degree of freedom. The phase is related with the SB Higgs as $\alpha=2 \pi g R\langle a\rangle(\bmod 2 \pi)$, where $g$ is the $5 \mathrm{D}$ gauge coupling and $a$ is a classical background of the extra dimensional component of the gauge fields, namely the SB Higgs. Some of the non-SB Higgs scalars also have interaction between the SB Higgs in the tree-level potential and have masses of $\mathcal{O}(\alpha / R)$. Since the K-K modes should be sufficiently heavy, a realistic vacuum satisfies $\alpha \ll 1$ and such a vacuum is dynamically realized with an appropriate choice of the bulk matter fields as discussed in the literature [10].

The SB Higgs has no mass term in the tree-level potential; the mass arises from the oneloop effective potential. The SB Higgs tends to be lighter than the massive gauge fields due to the loop suppression factor, which is inconsistent with the experimental bounds of Higgs searches [16]. A phenomenologically viable SB Higgs mass, however, can be realized through enhancement mechanism, as shown in [11, 12, 17].

The loop corrections to the masses of the non-SB Higgs have not been frequently discussed in the gauge-Higgs unification. The large one-loop mass correction to the SB Higgs mass 
implies that there also appear $\mathcal{O}\left(M_{W}\right)$ or larger one-loop contributions to the non-SB Higgs masses. The corrections are not suppressed rather than the tree-level masses of the non-SB Higgs, and thus are expected to give important effects on the multi-Higgs mass spectrum. In order to see this explicitly, we examine the one-loop corrections and multi-Higgs mass spectrum in a simple model.

\section{Multi-Higgs mass spectrum in a 5D SUSY model}

\subsection{Setup}

In this section, we consider a 5D SUSY gauge theory, where the fifth dimension is assumed to be compactified on the orbifold $S^{1} / Z_{2}$ and a multi-Higgs spectrum at low energy is predicted. On the orbifold, a point of the fifth dimensional coordinate $y$ is identified with other points by the translation, $U[y+2 \pi R] \sim y$, and the reflection, $P_{0}[-y] \sim y$. Combining them, one can define another reflection operator $P_{1} \equiv U^{-1} P_{0}$, then $\pi R-y$ and $\pi R+y$ are identified by the reflection $P_{1}[\pi R-y]=\pi R+y$. Since the points $y=0, \pi R$ are invariant under the reflections respectively, they are called orbifold fixed points.

The vector multiplet $\mathcal{V}$ in the $5 \mathrm{D}$ SUSY theory can be decomposed into a $4 \mathrm{D}$ chiral superfield $\Phi$ and a real vector superfield $V$ as follows:

$$
\mathcal{V}=\left(A_{M}^{a}, \eta_{1}^{a}, \eta_{2}^{a}, \Sigma^{a}\right) \rightarrow\left\{\begin{array}{l}
V=\left(A_{\mu}^{a}, \eta_{1}^{a}\right), \\
\Phi=\left(\Sigma^{a}+i A_{y}^{a}, \eta_{2}^{a}\right),
\end{array}\right.
$$

where $M=(\mu, y)$ and $A_{M}^{a}, \eta_{1,2}^{a}$ and $\Sigma^{a}$ are the $5 \mathrm{D}$ gauge field, Majorana spinors and a real scalar, respectively [15]. The subscript $a$ denotes the index of the adjoint representation of gauge group. The Lagrangian of the 5D SUSY theory is given as follows [18]:

$$
\mathcal{L}_{\text {vec }}=\operatorname{Tr}\left[-\frac{1}{2} F_{M N} F^{M N}+\left(D_{M} \Sigma\right) D^{M} \Sigma+\bar{\lambda}_{i} i \Gamma^{M} D_{M} \lambda_{i}-g \bar{\lambda}_{i}\left[\Sigma, \lambda_{i}\right]\right],
$$

where $g$ is the five dimensional gauge coupling constant and the Gamma matrices are defined by $\left(\Gamma^{\mu}, \Gamma^{y}\right)=\left(\gamma^{\mu}, i \gamma^{5}\right)$. The field strength and the covariant derivatives are defined by

$$
F_{M N} \equiv \partial_{M} A_{N}-\partial_{N} A_{M}-i g\left[A_{M}, A_{N}\right], \quad D_{M} \phi \equiv \partial_{M} \phi-i g\left[A_{M}, \phi\right],
$$

where $\phi$ implies fields in the adjoint representation. Fermions $\lambda_{i}(i=1,2)$ are symplecticMajorana spinors; they are written by

$$
\lambda_{i}^{a}=\left(\begin{array}{c}
\eta_{i}^{a} \\
\epsilon_{i j}\left(i \sigma^{2}\right) \eta_{j}^{a *}
\end{array}\right)
$$


where $\epsilon_{i j}$ is antisymmetric with $\epsilon_{12}=1$ and $\sigma^{2}$ is the Pauli matrix in the spinor space. It is known that the Lagrangian (3.2) has a global symmetry, called $S U(2)_{R}$, and the symplecticMajorana spinors are transformed as doublets under the symmetry.

One can introduce bulk hypermultiplets in the theory. The Lagrangian is written as follows [18]:

$$
\begin{aligned}
\mathcal{L}_{\text {mat }}= & \left|D_{M} \phi_{i}\right|^{2}-g^{2} \phi_{i}^{\dagger} \Sigma^{2} \phi_{i}-\frac{g^{2}}{2} \sum_{a, m}\left(\phi_{i}^{\dagger}\left(\tau^{m}\right)_{i j} t_{\phi}^{a} \phi_{j}\right)^{2} \\
& +\bar{\psi}\left(i \Gamma^{M} D_{M}-g \Sigma\right) \psi-\left(i g \sqrt{2} \bar{\psi} \phi_{i} \lambda_{i}+\text { h.c. }\right), \\
\left(D_{M} \phi\right)^{\alpha}= & \left(\partial_{M} \delta^{\alpha \beta}-i g A_{M}^{a}\left(t_{\phi}^{a}\right)^{\alpha \beta}\right) \phi^{\beta},
\end{aligned}
$$

where $\phi_{i}(i=1,2)$ are complex scalars, $\psi=\left(\tilde{\phi}_{L}, \tilde{\phi}_{R}\right)^{T}$ is a Dirac spinor, $t_{\phi}^{a}$ is the representation matrix of $\phi$, and $\tau^{m}(m=1,2,3)$ are $S U(2)_{R}$ generators.

The geometry $S^{1} / Z_{2}$ requires us to choose the boundary conditions for the fields: each field can have non-trivial transformations under the translation $U$ and the reflections $P_{0}$ and $P_{1}$ in such a way that the Lagrangian is invariant. Let $\varphi(x, y)$ be a general field in a representation space of the symmetry of theory. Transformation law of the field is defined by

$$
\mathcal{L}\left[\varphi\left(x, \mathcal{T}_{i}[y]\right)\right] \equiv \mathcal{L}\left[\mathcal{U}_{\varphi}\left[\mathcal{T}_{i}\right] \varphi(x, y)\right]
$$

where $\mathcal{T}_{i}=\left\{U, P_{0}, P_{1}\right\}$. The operator $\mathcal{U}_{\varphi}\left[\mathcal{T}_{i}\right]$ acts on the field in its representation space. The transformations of the coordinate satisfy

$$
P_{0}^{2}=P_{1}^{2}=\mathbf{1}, \quad U P_{0} U=P_{0}, \quad P_{1}=U^{-1} P_{0},
$$

where 1 denotes the identity operation. Corresponding to (3.8), transformations of the field should be chosen to satisfy the following set of constraints in order to keep the consistency of the translation and parity operations:

$$
\mathcal{U}_{\varphi}\left[P_{0}\right]^{2}=\mathcal{U}_{\varphi}\left[P_{1}\right]^{2}=\mathbf{1}, \quad \mathcal{U}_{\varphi}[U] \mathcal{U}_{\varphi}\left[P_{0}\right] \mathcal{U}_{\varphi}[U]=\mathcal{U}_{\varphi}\left[P_{0}\right], \quad \mathcal{U}_{\varphi}\left[P_{1}\right]=\mathcal{U}_{\varphi}[U]^{-1} \mathcal{U}_{\varphi}\left[P_{0}\right]
$$

The transformation law is referred to as the boundary condition of each field [19]. Using the last equality of (3.9), one can read the boundary condition of the parity $P_{1}$ from those of $U$ and $P_{0}$. The gauge symmetry of the theory can be broken through non-trivial boundary conditions. It is also known that the remaining $\mathcal{N}=1 \mathrm{SUSY}$ is explicitly broken with twisted boundary conditions for the $S U(2)_{R}$ doublets and we adopt this mechanism, the so-called Scherk-Schwarz SUSY breaking [20]. 
In the following, we examine a simple toy model where the multi-Higgs scalars appear at low energy. The original gauge symmetry of the theory is assumed to be $S U(2)$; the symmetry is explicitly broken to $U(1)$ by the particular boundary conditions which satisfy (3.9): for the $5 \mathrm{D}$ vector multiplet, we take

$$
\begin{aligned}
A_{\mu}(y+2 \pi R) & =A_{\mu}(y), \quad A_{\mu}(-y)=\tau_{3} A_{\mu}(y) \tau_{3}, \\
A_{y}(y+2 \pi R) & =A_{y}(y), \quad A_{y}(-y)=-\tau_{3} A_{y}(y) \tau_{3}, \\
\Sigma(y+2 \pi R) & =\Sigma(y), \quad \Sigma(-y)=-\tau_{3} \Sigma(y) \tau_{3}, \\
\left(\begin{array}{l}
\eta_{1} \\
\eta_{2}
\end{array}\right)(y+2 \pi R) & =\left(\begin{array}{cc}
\cos (2 \pi \beta) & -\sin (2 \pi \beta) \\
\sin (2 \pi \beta) & \cos (2 \pi \beta)
\end{array}\right)\left(\begin{array}{l}
\eta_{1} \\
\eta_{2}
\end{array}\right)(y), \\
\left(\begin{array}{c}
\eta_{1} \\
\eta_{2}
\end{array}\right)(-y) & =\left(\begin{array}{c}
\tau_{3} \eta_{1} \tau_{3} \\
-\tau_{3} \eta_{2} \tau_{3}
\end{array}\right)(y),
\end{aligned}
$$

and for the hypermultiplets, we take

$$
\begin{aligned}
\left(\begin{array}{l}
\phi_{1} \\
\phi_{2}
\end{array}\right)(y+2 \pi R) & =\eta_{U}\left(\begin{array}{cc}
\cos (2 \pi \beta) & -\sin (2 \pi \beta) \\
\sin (2 \pi \beta) & \cos (2 \pi \beta)
\end{array}\right)\left(\begin{array}{l}
\phi_{1} \\
\phi_{2}
\end{array}\right)(y), \\
\left(\begin{array}{c}
\phi_{1} \\
\phi_{2}
\end{array}\right)(-y) & =\eta_{P}\left(\begin{array}{c}
\mathcal{T}_{\phi}\left[t^{3}\right] \phi_{1} \\
-\mathcal{T}_{\phi}\left[t^{3}\right] \phi_{2}
\end{array}\right)(y), \\
\left(\begin{array}{c}
\tilde{\phi}_{L} \\
\tilde{\phi}_{R}
\end{array}\right)(y+2 \pi R) & =\eta_{U}\left(\begin{array}{c}
\tilde{\phi}_{L} \\
\tilde{\phi}_{R}
\end{array}\right)(y), \\
\left(\begin{array}{c}
\tilde{\phi}_{L} \\
\tilde{\phi}_{R}
\end{array}\right)(-y) & =\eta_{P}\left(\begin{array}{c}
\mathcal{T}_{\phi}\left[t^{3}\right] \tilde{\phi}_{L} \\
-\mathcal{T}_{\phi}\left[t^{3}\right] \tilde{\phi}_{R}
\end{array}\right)(y),
\end{aligned}
$$

where $\tau_{3}\left(\mathcal{T}_{\phi}\left[t^{3}\right]\right)$ is the diagonal generator of the $S U(2)$ gauge symmetry in the fundamental representation (in the representation of $\phi$ ). Additional parities of each hypermultiplet are incorporated by $\eta_{U}$ and $\eta_{P}$; they must be 1 or -1 in order to satisfy the consistency conditions (3.9). As mentioned above, we introduce the Scherk-Schwarz SUSY breaking with a parameter $\beta$ in a general form: if $\sin (2 \pi \beta) \neq 0$, then the residual $\mathcal{N}=1$ SUSY is broken and there appears $\mathcal{O}(\beta / R)(\bmod 1 / R)$ mass splitting between bosonic and fermionic states in the theory [21]. With the boundary conditions (3.10) and (3.11), among the gauge bosons only $A_{\mu}^{3}$ has massless zero mode, and it corresponds to the $4 \mathrm{D}$ gauge boson of the residual $U(1)$ gauge symmetry.

There appear four real scalar zero-modes, that is, Higgs scalars from the 5D vector multiplet (3.1) under the boundary conditions (3.10). We regard them as classical backgrounds of the theory and take the substitution in the Lagrangian (3.2) and (3.6):

$$
A_{y}^{a=1,2}\left(x^{M}\right) \rightarrow A_{y}^{a=1,2}\left(x^{M}\right)+a_{a=1,2}, \quad \Sigma^{a=1,2}\left(x^{M}\right) \rightarrow \Sigma^{a=1,2}\left(x^{M}\right)+\sigma_{a=1,2},
$$


where $a_{a}$ and $\sigma_{a}$ are the classical backgrounds. Except for the backgrounds, any fields in the theory are referred to as fluctuations. Among the backgrounds, $a_{1}$ and $a_{2}$ have Wilson line phase degrees of freedom and evolve non-trivial VEVs through quantum corrections [14]. Using the residual $U(1)$ gauge transformation, one can freely rotate the direction of the VEVs in the field space spanned by $a_{1}$ and $a_{2}$. We take $a_{2}$ as the SB Higgs field in the analyses. Then, $a_{1}$ is eaten by the longitudinal mode of the zero-mode of the $U(1)$ gauge field after the symmetry breaking. The other physical Higgs modes, $\sigma_{1}$ and $\sigma_{2}$, are the non-SB Higgs.

With the substitution (3.12) in the Lagrangian (3.2), the square of the fifth dimensional covariant derivative of $\Sigma$ yields the classical potential of the theory:

$$
\begin{aligned}
V_{\text {tree }} & =\frac{g^{2}}{2} \sum_{a, a^{\prime}, c, c^{\prime}=1}^{2} \sum_{d=1}^{3} a_{a} a_{a^{\prime}} \sigma_{c} \sigma_{c^{\prime}} f^{a c d} f^{a^{\prime} c^{\prime} d} \\
& =\frac{g^{2}}{2}\left(-\sigma_{2} a_{1}+a_{2} \sigma_{1}\right)^{2},
\end{aligned}
$$

where $f^{a b c}$ is the structure constant of the $S U(2)$ gauge symmetry. If the SB-Higgs is expanded around a VEV as $a_{2}=\tilde{a}_{2}+\left\langle a_{2}\right\rangle$, then the potential involves mass term for $\sigma_{1}$, which corresponds to the Higgs scalar associated with the non-flat direction of the potential. As mentioned above, $a_{1}$ is eaten by the longitudinal mode of the residual $U(1)$ gauge boson. The other backgrounds, $\tilde{a}_{2}$ and $\sigma_{2}$, are massless at the tree-level.

The backgrounds in (3.12) mix with each other through the residual $U(1)$ gauge transformation. It is useful to turn to a new basis where Higgs fields are eigenstates of the $U(1)$ gauge symmetry; with the following reparametrization, the classical backgrounds form a pair of complex scalars:

$$
n_{u}=\frac{1}{2}\left(i a_{1}+a_{2}+\sigma_{1}-i \sigma_{2}\right), \quad n_{d}=\frac{1}{2}\left(-i a_{1}+a_{2}-\sigma_{1}-i \sigma_{2}\right),
$$

where they have opposite charge of the $U(1)$ gauge symmetry. We refer to $n_{u, d}$ as up- and down-type Higgs scalars. In this basis, the tree-level potential (3.13) takes a clearer form as

$$
\begin{aligned}
V_{\text {tree }} & =\frac{g^{2}}{2}\left(\left|n_{u}\right|^{2}-\left|n_{d}\right|^{2}\right)^{2} \\
& =\frac{g^{2}}{2} D^{2} \\
D & \equiv-\sigma_{2} a_{1}+a_{2} \sigma_{1}=\left|n_{u}\right|^{2}-\left|n_{d}\right|^{2} .
\end{aligned}
$$

The form of the tree-level potential is constrained by the symmetries of the theory: it corresponds to the D-term potential of the residual $U(1)$ gauge symmetry in terms of $\mathcal{N}=1$ SUSY theory [15]. The flat directions lie along $D=0$. If a linear term of $D$ is incorporated in the theory, non-zero VEV of the D-term is realized and the SUSY is spontaneously broken [22]. 


\subsection{Perturbative evaluation of the effective potential}

In this subsection, we estimate the one-loop effective potential including all the modes of the Higgs scalars. The calculation is carried out with a straightforward way; at first we adopt K-K mode decomposition of all the fluctuations, and the fifth-dimensional coordinate is integrated out in the action. Then, we obtain the effective 4D theory and can estimate the functional integral with infinite towers of the K-K modes. As a result, contributions to the effective potential generally written by

$$
\begin{aligned}
\delta V= & \frac{-i}{2 \pi R} \frac{N_{B}}{2} \sum_{\mathrm{K}-\mathrm{K}} \ln \operatorname{det}\left[\Delta_{0 B}+m_{B} \Delta_{1 B}+\Delta_{2 B}\right] \\
& +\frac{i}{2 \pi R} \frac{N_{F}}{2} \sum_{\mathrm{K}-\mathrm{K}} \ln \operatorname{det}\left[\Delta_{0 F}+m_{F} \Delta_{1 F}+\Delta_{2 F}\right],
\end{aligned}
$$

where the determinants are taken over the $4 \mathrm{D}$ momentum space and representation space of the $S U(2)$ gauge symmetry. The first (second) line corresponds to the contributions from bosonic (fermionic) fluctuations. In the determinants, we take $\Delta_{0 B, F}=\left(\square+m_{B, F}^{2}\right) \cdot \mathbf{1}$ such that $m_{B, F}^{2}$ are independent of both the gauge coupling and the classical backgrounds, where $\mathbf{1}$ means identity matrix in representation space of the fluctuation fields in internal loops. The operators, $\Delta_{1}$ and $\Delta_{2}$, are of order $g^{1}$ and $g^{2}$, respectively. They also take forms of matrices in

specific representation space. The overall factor, $N_{B(F)}$, counts the bosonic (fermionic) degree of freedom in the internal loops.

If the eigenvalues of the operators are analytically obtained, then the functional determinant and the K-K mode summation may be directly evaluated. This is actually the case where one focuses only on a particular background of Wilson line phase degrees of freedom and the other backgrounds are set to zero. When one chooses $a_{2} \neq 0$ and $a_{1}=\sigma_{1}=\sigma_{2}=0$ for instance, a part of the contribution typically evaluated as follows [10, 11, 14, 17, 23, 24]:

$$
\frac{1}{2 \pi R} \int \frac{d^{4} p_{E}}{(2 \pi)^{4}} \sum_{n=-\infty}^{\infty} \ln \left[p_{E}^{2}+\left(\frac{n+g R a_{2}}{R}\right)^{2}\right]=\frac{3}{64 \pi^{7} R^{5}} \sum_{w=1}^{\infty} \frac{\cos \left(2 \pi g R w a_{2}\right)}{w^{5}}
$$

where $p_{E}$ is the Wick-rotated momentum and the background independent term is discarded in the right-hand side. The Wilson line phase degree of freedom $\alpha \equiv 2 \pi g R a_{2}(\bmod 2 \pi)$ appears in the cosine function. For a small value of $\alpha \ll 1$, K-K modes are sufficiently heavier than the weak scale, and one can approximately evaluate the summation as

$$
\sum_{w=1}^{\infty} \frac{\cos (\alpha w)}{w^{5}} \simeq \zeta(5)-\frac{\zeta(3)}{2} \alpha^{2}+\frac{\alpha^{4}}{288}\left(25-6 \log \left[\alpha^{2}\right]\right)+\mathcal{O}\left(\alpha^{6}\right)
$$


where $\zeta(s)$ is the Riemann zeta-function. The approximate expansion have a logarithmic singularity with $\alpha \rightarrow 0$; it implies the infra-red (IR) divergence of the zero-mode propagator in internal loops. A finite $\mathrm{VEV}$ of $\alpha$ gives non-zero masses of the zero-modes and thus the singularity disappears.

When one includes the general background fields (3.12), it is difficult to obtain all the eigenvalues and/or to carry out the summation as above. Here, we evaluate approximate forms of contributions to the effective potential as in (3.19). Functional determinants in (3.17) have a perturbative expansion of the gauge coupling and the background fields:

$$
\ln \operatorname{det}\left[\Delta_{0}+m \Delta_{1}+\Delta_{2}\right]=\ln \operatorname{det}\left[\Delta_{0}\right]+\sum_{f=1}^{\infty} \frac{(-1)^{f+1}}{f} \operatorname{Tr}\left[\left(\frac{m \Delta_{1}+\Delta_{2}}{\Delta_{0}}\right)^{f}\right]
$$

where $\Delta_{k}$ is $\mathcal{O}\left(\left(g n_{u, d}\right)^{k}\right)$. In the following analyses, we focus on the potential up to $\mathcal{O}\left(\left(g n_{u, d}\right)^{4}\right)$ and the higher-order corrections are neglected. This is valid if the typical energy scale of the classical backgrounds are much smaller than the compactification scale, namely $2 \pi R g n_{u, d} \ll 1$, which is consistent with phenomenological constraints as argued. In this case, it is sufficient to estimate the first few terms of the Taylor-expansion (3.20) as long as there is no IR singularity of the propagators in the internal loops. The IR singularities, $\Delta_{0}=0$, generally exist only in the contributions from zero-mode loops, and thus one needs to carry out the summation in (3.20). With this in mind, we perform a perturbative expansion of the functional determinant up to $\mathcal{O}\left(\left(g n_{u, d}\right)^{4}\right)$ as follows:

$$
\begin{gathered}
\ln \operatorname{det}\left[\Delta_{0}+m \Delta_{1}+\Delta_{2}\right] \\
=\ln \operatorname{det}\left[\Delta_{0}\right]+\operatorname{Tr}\left[\left(\frac{\Delta_{2}}{\Delta_{0}}\right)-\frac{1}{2}\left(\frac{m^{2} \Delta_{1}^{2}}{\Delta_{0}^{2}}\right)-\frac{1}{2}\left(\frac{\Delta_{2}^{2}}{\Delta_{0}^{2}}\right)+\left(\frac{m^{2} \Delta_{1}^{2} \Delta_{2}}{\Delta_{0}^{3}}\right)-\frac{1}{4}\left(\frac{m^{4} \Delta_{1}^{4}}{\Delta_{0}^{4}}\right)\right] \\
+(\operatorname{IR~div.~})+\mathcal{O}\left(\left(g n_{u, d}\right)^{5}\right) \\
=\ln \operatorname{det}\left[\Delta_{0}\right]+i \int \frac{d^{4} p_{E}}{(2 \pi)^{4}}\left\{\frac{1}{\left[p_{E}^{2}+m^{2}\right]} \operatorname{Tr}\left[\Delta_{2}\right]+\frac{m^{2}}{\left[p_{E}^{2}+m^{2}\right]^{2}} \operatorname{Tr}\left[-\frac{1}{2} \Delta_{1}^{2}\right]\right. \\
\left.+\frac{1}{\left[p_{E}^{2}+m^{2}\right]^{2}} \operatorname{Tr}\left[-\frac{1}{2} \Delta_{2}^{2}\right]+\frac{m^{2}}{\left[p_{E}^{2}+m^{2}\right]^{3}} \operatorname{Tr}\left[\Delta_{1}^{2} \Delta_{2}\right]+\frac{m^{4}}{\left[p_{E}^{2}+m^{2}\right]^{4}} \operatorname{Tr}\left[-\frac{1}{4} \Delta_{1}^{4}\right]\right\} \\
+(\operatorname{IR~div.~})+\mathcal{O}\left(\left(g n_{u, d}\right)^{5}\right),
\end{gathered}
$$

where possible contributions from the IR divergences are implied. In the present case, terms with odd order of $\Delta_{1}$ have odd orbifold parity and vanish. To proceed the perturbative calculation, one should reveal explicit forms of the operators in the functional determinant of each K-K mode. 


\subsubsection{One-loop correction from the vector multiplet}

We start to evaluate the one-loop correction from the 5D vector multiplet. As argued, we derive the effective 4D Lagrangian and then the functional integral is performed with the perturbative expansion (3.21). Let us introduce a gauge fixing function:

$$
G^{a}=\frac{1}{\sqrt{\xi}}\left[\partial^{\mu} \delta^{a c} A_{\mu}^{c}-\xi\left(\left[\partial_{y} \delta^{a c}+g f^{a b c} a_{b}\right] A_{y}^{c}+g f^{a b c} \sigma_{b} \Sigma^{c}\right)\right],
$$

where $\xi$ is a gauge parameter. The gauge fixing is regarded as an extension of the well-known $4 \mathrm{D} R_{\xi}$ gauge; gauge fixing terms and ghost Lagrangian are thus introduced as

$$
\mathcal{L}_{g f}=-\frac{1}{2} G^{c} G^{c}, \quad \mathcal{L}_{g h}=g \sqrt{\xi} \bar{c}^{a}\left[\frac{\delta G^{a}}{\delta \alpha^{c}}\right] c^{c} .
$$

Now the quadratic parts of the Lagrangian with respect to the fluctuations are written by

$$
\begin{aligned}
\left.\mathcal{L}_{\text {vec }}\right|_{\text {quadratic }}= & -\frac{1}{2} A_{\mu}^{a}\left(\mathcal{D}_{A_{\mu}}^{\mu \nu}\right)^{a c} A_{\nu}^{c}+\bar{c}^{a}\left(\mathcal{D}_{c}\right)^{a c} c^{c}+\frac{1}{2} A_{y}^{a}\left(\mathcal{D}_{A_{y}}\right)^{a c} A_{y}^{c}+\frac{1}{2} \Sigma^{a}\left(\mathcal{D}_{\Sigma}\right)^{a c} \Sigma^{c} \\
& +\frac{i}{2} \bar{\lambda}_{1}^{a}\left(\mathcal{D}_{\lambda}\right)^{a c} \lambda_{1}^{c}+\frac{i}{2} \bar{\lambda}_{2}^{a}\left(\mathcal{D}_{\lambda}\right)^{a c} \lambda_{2}^{c}+\mathcal{L}_{\text {others }} .
\end{aligned}
$$

In the above expression, the background dependent operators $(\mathcal{D})$ are written by

$$
\begin{aligned}
\left(\mathcal{D}_{A_{\mu}}^{\mu \nu}\right)^{a c} & =\left(-\left[\square-\partial_{y}^{2}\right] \delta^{a c}+2 g f^{a b c} a_{b} \partial_{y}+g^{2} f^{a b d} f^{d b^{\prime} c}\left(a_{b} a_{b^{\prime}}+\sigma_{b} \sigma_{b^{\prime}}\right)\right) \eta^{\mu \nu}+\partial^{\mu} \partial^{\nu}\left(1-\xi^{-1}\right) \delta^{a c}, \\
\left(\mathcal{D}_{c}\right)^{a c} & =-\left[\square-\xi \partial_{y}^{2}\right] \delta^{a c}+2 g \xi f^{a b c} a_{b} \partial_{y}+\xi g^{2} f^{a b d} f^{d b^{\prime} c}\left(a_{b} a_{b^{\prime}}+\sigma_{b} \sigma_{b^{\prime}}\right), \\
\left(\mathcal{D}_{A_{y}}\right)^{a c} & =-\left[\square-\xi \partial_{y}^{2}\right] \delta^{a c}+2 g \xi f^{a b c} a_{b} \partial_{y}+g^{2} f^{a b d} f^{d b^{\prime} c}\left(\xi a_{b} a_{b^{\prime}}+\sigma_{b} \sigma_{b^{\prime}}\right), \\
\left(\mathcal{D}_{\Sigma}\right)^{a c} & =-\left[\square-\partial_{y}^{2}\right] \delta^{a c}+2 g f^{a b c} a_{b} \partial_{y}+g^{2} f^{a b d} f^{d b^{\prime} c}\left(a_{b} a_{b^{\prime}}+\xi \sigma_{b} \sigma_{b^{\prime}}\right), \\
\left(\mathcal{D}_{\lambda}\right)^{a c} & =\Gamma^{M} \partial_{M} \delta^{a c}-g f^{a b c}\left(i \gamma^{5} a_{b}+\sigma_{b}\right),
\end{aligned}
$$

where $\square$ denotes the four dimensional D'Alambertian operator. In (3.24), mixing between fluctuations $A_{y}$ and $\Sigma$ appears as follows:

$$
\mathcal{L}_{\text {others }}=g(\xi-1) A_{y}^{a} f^{a b c} \sigma_{b} \partial_{y} \Sigma^{c}-g^{2} A_{y}^{a}\left[f^{a c d} f^{d b b^{\prime}} a_{b} \sigma_{b^{\prime}}+f^{a b d} f^{d b^{\prime} c}\left(\sigma_{b} a_{b^{\prime}}-\xi a_{b} \sigma_{b^{\prime}}\right)\right] \Sigma^{c} .
$$

The above forms are simplified with $\xi=1$ and we adopt this specific choice of gauge fixing in the analyses $\S$

\footnotetext{
$\S$ A factor in (3.26) was misread in ref. 14; the results of the analysis do not depend on the factor when one focuses only on the particular backgrounds of flat directions.
} 
With the boundary conditions (3.10), K-K decomposition of the fields in the vector multiplets is written as follows:

$$
\begin{aligned}
& \left(\begin{array}{c}
A_{\mu}^{3}(x, y) \\
A_{y}^{1,2}(x, y) \\
\Sigma^{1,2}(x, y)
\end{array}\right)=\frac{1}{\sqrt{2 \pi R}}\left(\begin{array}{c}
\left(A_{\mu}^{(0)}\right)^{3}(x) \\
\left(A_{y}^{(0)}\right)^{1,2}(x) \\
\left(\Sigma^{(0)}\right)^{1,2}(x)
\end{array}\right)+\frac{1}{\sqrt{\pi R}} \sum_{n=1}^{\infty}\left(\begin{array}{c}
\left(A_{\mu}^{(n)}\right)^{3}(x) \\
\left(A_{y}^{(n)}\right)^{1,2}(x) \\
\left(\Sigma^{(n)}\right)^{1,2}(x)
\end{array}\right) \cos \left(\frac{n y}{R}\right), \\
& \left(\begin{array}{c}
A_{\mu}^{1,2}(x, y) \\
A_{y}^{3}(x, y) \\
\Sigma^{3}(x, y)
\end{array}\right)=\frac{1}{\sqrt{\pi R}} \sum_{n=1}^{\infty}\left(\begin{array}{c}
\left(A_{\mu}^{(n)}\right)^{1,2}(x) \\
\left(A_{y}^{(n)}\right)^{3}(x) \\
\left(\Sigma^{(n)}\right)^{3}(x)
\end{array}\right) \sin \left(\frac{n y}{R}\right) \\
& \left(\begin{array}{l}
\eta_{1}^{1,2}(x, y) \\
\eta_{2}^{1,2}(x, y)
\end{array}\right)=\frac{1}{\sqrt{\pi R}}\left(\begin{array}{cc}
\cos \left(\frac{\beta y}{R}\right) & -\sin \left(\frac{\beta y}{R}\right) \\
\sin \left(\frac{\beta y}{R}\right) & \cos \left(\frac{\beta y}{R}\right)
\end{array}\right) \\
& \times\left(\left(\begin{array}{c}
0 \\
2^{-1 / 2}\left(\eta_{2}^{(0)}\right)^{1,2}(x)
\end{array}\right)+\sum_{n=1}^{\infty}\left(\begin{array}{l}
\left(\eta_{1}^{(n)}\right)^{1,2}(x) \sin \left(\frac{n y}{R}\right) \\
\left(\eta_{2}^{(n)}\right)^{1,2}(x) \cos \left(\frac{n y}{R}\right)
\end{array}\right)\right), \\
& \left(\begin{array}{l}
\eta_{1}^{3}(x, y) \\
\eta_{2}^{3}(x, y)
\end{array}\right)=\frac{1}{\sqrt{\pi R}}\left(\begin{array}{cc}
\cos \left(\frac{\beta y}{R}\right) & -\sin \left(\frac{\beta y}{R}\right) \\
\sin \left(\frac{\beta y}{R}\right) & \cos \left(\frac{\beta y}{R}\right)
\end{array}\right) \\
& \times\left(\left(\begin{array}{c}
2^{-1 / 2}\left(\eta_{1}^{(0)}\right)^{3}(x) \\
0
\end{array}\right)+\sum_{n=1}^{\infty}\left(\begin{array}{c}
\left(\eta_{1}^{(n)}\right)^{3}(x) \cos \left(\frac{n y}{R}\right) \\
\left(\eta_{2}^{(n)}\right)^{3}(x) \sin \left(\frac{n y}{R}\right)
\end{array}\right)\right) .
\end{aligned}
$$

Using the K-K decomposition, one can easily carry out the $y$-integral in the action from $y=0$ to $y=2 \pi R$. Then, the obtained $4 \mathrm{D}$ Lagrangian includes infinite towers of $\mathrm{K}-\mathrm{K}$ modes in addition to the zero modes.

One can readily derive the contribution to the effective potential from the quadratic terms of the $4 \mathrm{D}$ effective Lagrangian. The contribution depends on the SUSY breaking parameter $\beta$, and is written by

$$
\begin{aligned}
\delta V_{\text {vec }}(\beta)= & \frac{-i}{2 \pi R} \frac{4-2}{2} \ln \operatorname{det}\left[\Delta_{0}\left(A_{\mu}^{(0)}\right)+\Delta_{2}\left(A_{\mu}^{(0)}\right)\right] \\
& +\frac{-i}{2 \pi R} \frac{4-2}{2} \sum_{n=1}^{\infty} \ln \operatorname{det}\left[\Delta_{0}\left(A_{\mu}^{(n)}\right)+m_{A_{\mu}}^{(n)} \Delta_{1}\left(A_{\mu}^{(n)}\right)+\Delta_{2}\left(A_{\mu}^{(n)}\right)\right] \\
& +\frac{-i}{2 \pi R} \frac{2}{2} \ln \operatorname{det}\left[\Delta_{0}\left(Q^{(0)}\right)+\Delta_{2}\left(Q^{(0)}\right)\right] \\
& +\frac{-i}{2 \pi R} \frac{2}{2} \sum_{n=1}^{\infty} \ln \operatorname{det}\left[\Delta_{0}\left(Q^{(n)}\right)+m_{Q}^{(n)} \Delta_{1}\left(Q^{(n)}\right)+\Delta_{2}\left(Q^{(n)}\right)\right] \\
& +\frac{i}{2 \pi R} \frac{2}{2} \sum_{n=-\infty}^{\infty} \ln \operatorname{det}\left[\Delta_{0}\left(\lambda^{(n)}\right)+m_{\lambda}^{(n)} \Delta_{1}\left(\lambda^{(n)}\right)+\Delta_{2}\left(\lambda^{(n)}\right)\right]
\end{aligned}
$$

where $Q^{(n)} \equiv \Sigma^{(n)}+i A_{y}^{(n)}$, and the fermionic fluctuations are implied by $\lambda^{(n)}$. The operators $\Delta_{i}(\varphi)$ denotes the contribution from a fluctuation $\varphi$ to $\Delta_{i}$ and is defined in Appendix A. 
Masses of the bosonic K-K modes, $A_{\mu}^{(n)}$ and $Q^{(n)}$, are the same as $m_{A_{\mu}}^{(n)}=m_{Q}^{(n)}=n / R$, on the other hand, the fermionic fluctuations have mass corrections through the Scherk-Schwarz SUSY breaking. Non-zero modes of $\eta_{1}^{(n)}$ and $\eta_{2}^{(n)}$ mix with each other due to K-K masses arising from $y$-derivative in the kinetic terms; they are rearranged into mass eigenstates, which are formally written by $n \geq 1$ and $n \leq-1$ modes of the masses $m_{\lambda}^{(n)}=(\beta+n) / R$. Combining the zero-mode contributions, all the contributions from fermionic fluctuations are written by the summation over $-\infty \leq n \leq \infty$.

Let us now evaluate the contribution to the effective potential up to $\mathcal{O}\left(\left(g n_{u, d}\right)^{4}\right)$ via the perturbative expansion. In (3.27), we rewrite the functional determinant by the approximate form of (3.21) and obtain the expression as

$$
\begin{aligned}
\delta V_{\text {vec }}(\beta) \simeq & \frac{3}{2 \pi R} \sum_{n=-\infty}^{\infty} \ln \operatorname{det}\left[\left[\square+(n / R)^{2}\right]-\left[\square+\left(\frac{\beta+n}{R}\right)^{2}\right]\right] \\
& +\mathcal{F}_{[1,0, \beta]}\left(4 g^{2} S\right)+\mathcal{F}_{[2,1, \beta]}\left(-4 g^{2}(S+T)\right)+\mathcal{F}_{[2,0, \beta]}\left(-4 g^{4} S^{2}\right) \\
& +\mathcal{F}_{[3,1, \beta]}\left(4 g^{4}\left(4 S^{2}-D^{2}+4 S T\right)\right)+\mathcal{F}_{[4,2, \beta]}\left(-8 g^{4}\left(S^{2}+2 S T+T^{2}\right)\right) \\
& +\frac{1}{2 \pi R} \hat{\zeta}_{[2,0,0]}\left(-g^{4} D^{2}\right)+\frac{1}{2 \pi R} \int \frac{d^{4} p_{E}}{(2 \pi)^{4}} \frac{1}{\left[p_{E}^{2}\right]^{2}}\left(-2 g^{4} D^{2}\right) \\
& +\frac{1}{2 \pi R} \sum_{f=3}^{\infty} \frac{(-1)^{f+1}}{f} \int \frac{d^{4} p_{E}}{(2 \pi)^{4}} \frac{1}{\left[p_{E}^{2}\right]^{f}}\left(2 g^{2} S\right)^{f} \\
& +\frac{1}{2 \pi R} \sum_{f=3}^{\infty} \frac{(-1)^{f+1}}{f} \int \frac{d^{4} p_{E}}{(2 \pi)^{4}} \frac{1}{\left[p_{E}^{2}\right]^{f}} g^{2 f}\left[\left(S+\sqrt{S^{2}+3 D^{2}}\right)^{f}+\left(S-\sqrt{S^{2}+3 D^{2}}\right)^{f}\right] \\
& -\frac{2}{2 \pi R} \sum_{f=3}^{\infty} \frac{(-1)^{f+1}}{f} \int \frac{d^{4} p_{E}}{(2 \pi)^{4}} \frac{1}{\left[p_{E}^{2}\right]^{f}}\left[\left(2 g^{2} S\right)^{f}\right] \delta_{\beta, 0},
\end{aligned}
$$

where the Higgs fields $n_{u, d}$ are expressed in terms of the combinations

$$
S \equiv\left|n_{u}\right|^{2}+\left|n_{d}\right|^{2}, \quad T \equiv n_{u} n_{d}+\text { h.c. }
$$

and $D$ in (3.16). These combinations are invariant under the residual $U(1)$ gauge transformation. Loop functions including the K-K mode summation are defined by

$$
\begin{aligned}
\mathcal{F}_{[x, m, \beta]} & =\frac{1}{2 \pi R}\left[\hat{\zeta}_{[x, m, 0]}-\hat{\zeta}_{[x, m, \beta]}\right], \\
\hat{\zeta}_{[x, m, \beta]} & =\sum_{n=-\infty}^{\infty} \int \frac{d^{4} p_{E}}{(2 \pi)^{4}} \frac{\left(\frac{n+\beta}{R}\right)^{2 m}}{\left[p_{E}^{2}+\left(\frac{n+\beta}{R}\right)^{2}\right] x},
\end{aligned}
$$

where evaluation of the functions is shown in Appendix B. The last term in (3.28) is zero except for $\mathcal{N}=1$ SUSY limit, that is $\delta_{\beta \neq 0,0}=0$ and $\delta_{\beta=0,0}=1$, up to $\mathcal{O}\left(\left(g n_{u, d}\right)^{4}\right)$. 
Several points should be clarified in (3.28). The first line contributes to vacuum energy and is independent of the Higgs fields. We neglect the irrelevant constants hereafter. The second and third lines include the loop functions $\mathcal{F}_{[x, m, \beta]}$. As shown in Appendix $\mathbb{B}$, the loop integrals with $\mathrm{K}-\mathrm{K}$ mode summation can be divided into two parts: one is an UV divergent integral which respects the 5D Lorentz invariance and the other is a finite correction which violates the invariance. An observation is that the UV divergence does not depend on the parameter $\beta$ and thus respects SUSY. On the other hand, the finite corrections depend on the SUSY breaking effects. Hence, the divergent contributions from bosonic and fermionic fluctuations are canceled out in the functions $\mathcal{F}_{[x, m, \beta]}$, and only UV finite contributions remain.

The fourth line also includes loop integrals. The UV divergent contributions of the terms proportional to $D^{2}$ are found. The UV divergence of the first term is 5D Lorentz invariant and can be realized as a bulk term. On the other hand, the second term explicitly violates the $5 \mathrm{D}$ Lorentz symmetry and is considered as the divergence localized at the fixed points of the orbifold [25]. Again these UV divergences respect SUSY. Such the divergent contributions are known to exist in $S^{1} / Z_{2}$ exact SUSY theory and can be renormalized in a supersymmetric fashion [26]. We here focus on the SUSY breaking contributions; the divergence are simply subtracted and a regularized quantity is defined by $\Delta V_{\text {vec }} \equiv \delta V_{\text {vec }}(\beta)-\delta V_{\text {vec }}(0)$. Thereby, the one-loop corrections are written by finite contributions which break both SUSY and 5D Lorentz symmetry. $\|$

The last three lines of (3.28) are the contributions from possible IR divergent massless propagators of the zero-modes. With a suitable regularization of the worse IR behavior, one can extract the IR singularities and finite contributions to $\mathcal{O}\left(\left(g n_{u, d}\right)^{4}\right)$ terms. Notice that the IR singularities are also involved in $\hat{\zeta}_{[2,0,0]}$. Non-vanishing VEVs of the Higgs fields provide the physical cutoff of the IR divergences and the singularities are canceled out in the final expression as seen below.

Using the explicit evaluation of the loop functions in the Appendix B, one leads to the one-loop contribution to the effective potential. We focus on the case with $\beta \neq 0$, then the contribution is written as follows:

$$
\begin{aligned}
\Delta V_{\text {vec }}= & \frac{4 g^{2}}{64 \pi^{5} R^{3}} \sum_{w=1}^{\infty} \frac{4 \sin ^{2}(\pi w \beta)}{w^{3}}[2 S+T] \\
& +\frac{4 g^{4}}{3 \cdot 64 \pi^{3} R}\left\{-19 S^{2}-8 S T+2 T^{2}+3 D^{2}+6 S^{2} \ln \left[\frac{(2 \pi R)^{2} 2 g^{2} S}{4 \sin ^{2}(\pi \beta)}\right]\right\}
\end{aligned}
$$

\footnotetext{
I For $\sigma_{1}=\sigma_{2}=0$, the divergences disappear in each of the bosonic and fermionic contributions. The divergences are related to $\Sigma^{1,2}$ which are just scalar fields and are not protected if SUSY is not there.

I| Renormalization procedure may bring higher order contributions [13] and is left for future studies.
} 
where we discard the contribution to the vacuum energy as stated. The last logarithmic term includes the Higgs fields, so that a non-zero Higgs VEV correctly provides the physical cutoff of the IR divergence of the effective potential [27].

The result precisely reproduces the evaluation in the flat limit where the classical backgrounds are set zero except for a mode corresponding to a flat direction of the tree-level potential (3.15), namely the SB Higgs. One can choose $\alpha=2 \pi g R a_{2} \neq 0$ and $a_{1}=\sigma_{1}=\sigma_{2}=0$ for instance, then the one-loop potential takes

$$
\Delta V_{\text {vec }} \rightarrow \frac{-6}{64 \pi^{7} R^{5}}\left\{-\sum_{w=1}^{\infty} \frac{\sin ^{2}(\pi w \beta)}{w^{3}} \alpha^{2}+\frac{\alpha^{4}}{288}\left[25-6 \ln \left[\frac{\alpha^{2}}{4 \sin ^{2}(\pi \beta)}\right]\right]\right\} .
$$

This is actually realized as the expansion around $\alpha \ll \beta \lesssim 1$ up to $\mathcal{O}\left(\alpha^{4}\right)$ of the well-known one-loop correction [23, 24]:

$$
\Delta V_{\text {vec }}^{(\text {flat })}=\frac{-6}{64 \pi^{7} R^{5}} \sum_{w=1}^{\infty} \frac{\cos (\alpha w)}{w^{5}}(1-\cos (2 \pi w \beta)) .
$$

\subsubsection{One-loop correction from bulk hypermultiplets}

Let us discuss loop corrections from bulk hypermultiplets. Since the evaluation is carried out in a similar way as the vector multiplet, we briefly summarize the result here. For simplicity, we only introduce the fundamental representation in the analyses; generalization to the other representation is straightforward.

Since the hypermultiplets may involve chiral fermions in their zero-modes, a gauge anomaly of the residual $U(1)$ gauge symmetry generally appears [28]. The zero-mode contents depend on the parities $\eta_{U, P}$ in the boundary conditions (3.11). Non-zero contribution to the anomaly actually emerges from the loop effects of a massless chiral fermion in the $\eta_{U}=+1$ hypermultiplet. To evade the awkward case naively, we introduce $N_{+}$pairs of $\left(\eta_{U}, \eta_{P}\right)=(+1,+1)$ and $\left(\eta_{U}, \eta_{P}\right)=(+1,-1)$ multiplets in the following analyses. Then, the fermion zero-modes always form vector-like pairs and the anomaly is canceled out in the contributions from each pair ** For $\eta_{U}=-1$, though there are no massless zero-modes, anomalies localized at both the fixed points with opposite sign are induced. They are canceled out after the $y$-integration, but cause inconsistency in the full 5D theory without the help of some cancellation mechanisms. We here simply introduce $N_{-}$pairs of $\left(\eta_{U}, \eta_{P}\right)=(-1,+1)$ and $(-1,-1)$ to cancel the anomalies, as the above treatment of $\eta_{U}=1$.

\footnotetext{
** A divergent tadpole of the residual $U(1)$ D-term, which is known to localize at the orbifold fixed point [28, 29, 30, is found in each loop contribution from the hypermultiplets. The tadpole contributions from $\left(\eta_{U}, \eta_{P}\right)=$ $(+1,+1)$ and $\left(\eta_{U}, \eta_{P}\right)=(+1,-1)$ hypermultiplets have opposite sign and are canceled out as the anomaly.
} 
With the parity assignments, one can derive K-K expansion from (3.11) and 4D effective theory is obtained by carrying out the $y$-integration of the 5D Lagrangian (3.6), where the substitution (3.12) is understood. The quadratic terms of the fluctuations in the Lagrangian yields one-loop contributions to the effective potential from the bulk hypermultiplets:

$$
\begin{aligned}
\delta V_{\text {hyp }}(\beta)= & \frac{-i}{2 \pi R} \frac{2 \cdot 2 N_{+}}{2} \sum_{n=-\infty}^{\infty} \ln \operatorname{det}\left[\Delta_{0}\left(\phi_{+}^{(n)}\right)+m_{\phi_{+}}^{(n)} \Delta_{1}\left(\phi^{(n)}\right)+\Delta_{2}\left(\phi^{(n)}\right)\right] \\
& +\frac{i}{2 \pi R} \frac{4 N_{+}}{2}\left[\ln \operatorname{det}\left[\Delta_{0}\left(\psi^{(0)}\right)+\Delta_{2}\left(\psi_{u}^{(0)}\right)\right]+\ln \operatorname{det}\left[\Delta_{0}\left(\psi^{(0)}\right)+\Delta_{2}\left(\psi_{d}^{(0)}\right)\right]\right] \\
& +\frac{i}{2 \pi R} \frac{4 \cdot 2 N_{+}}{2} \sum_{n=1}^{\infty} \ln \operatorname{det}\left[\Delta_{0}\left(\psi_{+}^{(n)}\right)+m_{\psi_{+}}^{(n)} \Delta_{1}\left(\psi^{(n)}\right)+\Delta_{2}\left(\psi^{(n)}\right)\right] \\
& +\frac{-i}{2 \pi R} \frac{2 \cdot 2 N_{-}}{2} \sum_{n=-\infty}^{\infty} \ln \operatorname{det}\left[\Delta_{0}\left(\phi_{-}^{(n)}\right)+m_{\phi_{-}}^{(n)} \Delta_{1}\left(\phi^{(n)}\right)+\Delta_{2}\left(\phi^{(n)}\right)\right] \\
& +\frac{i}{2 \pi R} \frac{4 \cdot 2 N_{-}}{2} \sum_{n=1}^{\infty} \ln \operatorname{det}\left[\Delta_{0}\left(\psi_{-}^{(n)}\right)+m_{\psi_{-}}^{(n)} \Delta_{1}\left(\psi^{(n)}\right)+\Delta_{2}\left(\psi^{(n)}\right)\right]
\end{aligned}
$$

where $\phi(\psi)$ implies the bosonic (fermionic) fluctuations. The operators $\Delta_{i}(\varphi)$ and the masses $m_{\varphi}^{(n)}$ in the functional determinants are listed in Appendix $\mathrm{A}$ Note that, in contrast to the vector multiplet, bosonic fluctuations have the Scherk-Schwarz SUSY breaking masses in the hypermultiplets. Non-zero modes of $\phi_{1}$ and $\phi_{2}$ mix with each other, and then the contributions from the bosonic fluctuations are collected into the summation over $-\infty \leq n \leq \infty$.

The functional determinants are evaluated in the same way as the case of the vector multiplet. With a non-zero value of $\beta$, the one-loop contribution up to $\mathcal{O}\left(\left(g n_{u, d}\right)^{4}\right)$ is obtained as

$$
\begin{aligned}
\Delta V_{\text {hyp }}= & \frac{-2 N_{+} g^{2}}{64 \pi^{5} R^{3}} \sum_{w=1}^{\infty} \frac{4 \sin ^{2}(\pi w \beta)}{w^{3}}[2 S+T] \\
& +\frac{-2 N_{+} g^{4}}{12 \cdot 64 \pi^{3} R}\left\{-19 S^{2}-8 S T+2 T^{2}+6 S^{2} \ln \left[\frac{(2 \pi R)^{2} g^{2} S / 2}{4 \sin ^{2}(\pi \beta)}\right]\right\} \\
& +\frac{-2 N_{-} g^{2}}{64 \pi^{5} R^{3}} \sum_{w=1}^{\infty} \frac{4(-1)^{w} \sin ^{2}(\pi w \beta)}{w^{3}}(2 S+T)+\frac{N_{-} g^{4} S^{2}}{64 \pi^{3} R} \ln \left[\cos ^{2}(\pi \beta)\right] .
\end{aligned}
$$

${ }^{\dagger \dagger}$ In the present case, eigenvalues of the operators are analytically obtained due to the simpleness of the $S U(2)$ fundamental representation. Thus, one may try to directly evaluate the functional determinant rather than the perturbative calculation (3.21). It is, however, difficult to carry out the K-K mode resummation. 
As the case with vector multiplet, the flat limit is taken as

$$
\begin{aligned}
\Delta V_{\text {hyp }} \rightarrow & \frac{6 \cdot 2 N_{+}}{64 \pi^{7} R^{5}}\left[-\sum_{w=1}^{\infty} \frac{\sin ^{2}(\pi w \beta)}{w^{3}}(\alpha / 2)^{2}+\frac{(\alpha / 2)^{4}}{288}\left\{25-6 \ln \left[\frac{(\alpha / 2)^{2}}{4 \sin ^{2}(\pi \beta)}\right]\right\}\right] \\
& +\frac{6 \cdot 2 N_{-}}{64 \pi^{7} R^{5}}\left[-\sum_{w=1}^{\infty} \frac{(-1)^{w} \sin ^{2}(\pi w \beta)}{w^{3}}(\alpha / 2)^{2}+\frac{(\alpha / 2)^{4}}{48} \ln \left[\cos ^{2}(\pi \beta)\right]\right]
\end{aligned}
$$

This corresponds to the expansion of the known forms of the following potential around $\alpha \ll \beta \lesssim 1$ up to $\mathcal{O}\left(\alpha^{4}\right)$ [23, 24]:

$$
\begin{aligned}
\Delta V_{\text {hyp }}^{(\mathrm{flat})}= & \frac{6 \cdot 2 N_{+}}{64 \pi^{7} R^{5}} \sum_{w=1}^{\infty} \frac{\cos (\alpha w / 2)}{w^{5}}(1-\cos (2 \pi w \beta)) \\
& +\frac{6 \cdot 2 N_{-}}{64 \pi^{7} R^{5}} \sum_{w=1}^{\infty} \frac{\cos ((\alpha / 2-\pi) w)}{w^{5}}(1-\cos (2 \pi w \beta)) .
\end{aligned}
$$

\subsection{One-loop Higgs potential and mass spectrum}

We examined the bulk loop contributions to the effective potential in the previous subsection. Then, the approximate forms of the potential (3.31) and (3.35), which involve all the background of the scalar zero-modes, are explicitly obtained up to $\mathcal{O}\left(\left(g n_{u, d}\right)^{4}\right)$. The potential should be regarded as low-energy effective Higgs potential. In this subsection, we proceed to study the vacuum and mass spectrum using the effective potential.

In order to make the discussion clear, we introduce the $4 \mathrm{D}$ normalization as

$$
\begin{array}{r}
g_{c}=g L^{-1 / 2}, \quad h_{u}=n_{u} L^{1 / 2}, \quad h_{d}=n_{d} L^{1 / 2} \\
V_{h}^{0}=L V_{\text {tree }}, \quad V_{h}^{1}=L\left(\Delta V_{\text {vec }}+\Delta V_{\text {hyp }}\right)
\end{array}
$$

where $L=2 \pi R, g_{c}$ is $4 \mathrm{D}$ dimensionless gauge coupling and $h_{u, d}$ are up- and down-type Higgs scalars in the canonical normalization. From (3.15), (3.31) and (3.35), the Higgs potential is derived as follows:

$$
\begin{aligned}
V_{h}^{0}= & \frac{g_{c}^{2}}{2}\left(\left|h_{u}\right|^{2}-\left|h_{d}\right|^{2}\right), \\
V_{h}^{1}= & \frac{2 g_{c}^{2}}{\pi^{2}} \frac{1}{(2 \pi R)^{2}}\left[\left(1-\frac{N_{+}}{2}\right) \mathcal{C}_{\beta}^{+}-\frac{N_{-}}{2} \mathcal{C}_{\beta}^{-}\right]\left[2\left(\left|h_{u}\right|^{2}+\left|h_{d}\right|^{2}\right)+\left(h_{u} h_{d}+\text { h.c. }\right)\right] \\
& +\frac{g_{c}^{4}}{24 \pi^{2}}\left\{-19\left(\left|h_{u}\right|^{2}+\left|h_{d}\right|^{2}\right)^{2}-8\left(\left|h_{u}\right|^{2}+\left|h_{d}\right|^{2}\right)\left(h_{u} h_{d}+\text { h.c. }\right)+2\left(h_{u} h_{d}+\text { h.c. }\right)^{2}\right. \\
& \left.+3\left(\left|h_{u}\right|^{2}-\left|h_{d}\right|^{2}\right)^{2}+6\left(\left|h_{u}\right|^{2}+\left|h_{d}\right|^{2}\right)^{2} \ln \left[\frac{\left(\left|h_{u}\right|^{2}+\left|h_{d}\right|^{2}\right)}{\mathcal{C}_{\beta}^{\ell}}\right]\right\}
\end{aligned}
$$




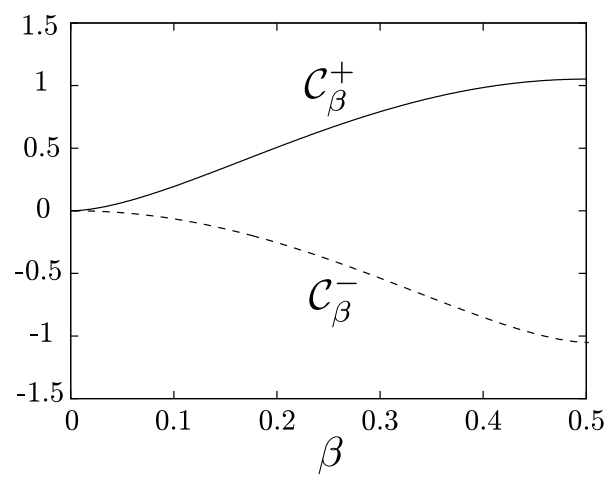

Figure 1: The values of $\mathcal{C}_{\beta}^{+}$and $\mathcal{C}_{\beta}^{-}$as the functions of $\beta$.

$$
\begin{aligned}
& -\frac{N_{+}}{8} \frac{g_{c}^{4}}{24 \pi^{2}}\left\{-19\left(\left|h_{u}\right|^{2}+\left|h_{d}\right|^{2}\right)^{2}-8\left(\left|h_{u}\right|^{2}+\left|h_{d}\right|^{2}\right)\left(h_{u} h_{d}+\text { h.c. }\right)+2\left(h_{u} h_{d}+\text { h.c. }\right)^{2}\right. \\
& \left.+6\left(\left|h_{u}\right|^{2}+\left|h_{d}\right|^{2}\right)^{2} \ln \left[\frac{\left(\left|h_{u}\right|^{2}+\left|h_{d}\right|^{2}\right)}{4 \mathcal{C}_{\beta}^{\ell}}\right]\right\} \\
& +\frac{6 N_{-}}{8} \frac{g_{c}^{4}}{24 \pi^{2}} \ln \left[\cos ^{2}(\pi \beta)\right]\left(\left|h_{u}\right|^{2}+\left|h_{d}\right|^{2}\right)^{2}
\end{aligned}
$$

where

$$
\mathcal{C}_{\beta}^{+}=\sum_{w=1}^{\infty} \frac{\sin ^{2}(\pi w \beta)}{w^{3}}, \quad \mathcal{C}_{\beta}^{-}=\sum_{w=1}^{\infty} \frac{(-1)^{w} \sin ^{2}(\pi w \beta)}{w^{3}}, \quad \mathcal{C}_{\beta}^{\ell}=\frac{2 \sin ^{2}(\pi \beta)}{(2 \pi R)^{2} g_{c}^{2}} .
$$

The numerical factors $\mathcal{C}_{\beta}^{+}$and $\mathcal{C}_{\beta}^{-}$are displayed in fig. 1 as the functions of $\beta$.

The effective potential (3.38) is invariant under the replacement between $h_{u}$ and $h_{d}$. Therefore, the field space which satisfies $h_{u}=h_{d}$ always becomes stationary against the variation corresponding to the mode of the non-flat direction, $h_{u} \neq h_{d}$. We thus focus on minima along the flat direction in the present analyses; if there is no tachyonic mode around the minima, the stability of the minima is locally ensured. The Higgs scalars are expanded around the minimum as

$$
h_{u}=\frac{1}{\sqrt{2}}\left(v_{h}+r_{u}+i \pi_{u}\right), \quad h_{d}=\frac{1}{\sqrt{2}}\left(v_{h}+r_{d}+i \pi_{d}\right)
$$

where $v_{h}$ is the Higgs VEV taken to be real positive, and $r_{u, d}$ and $\pi_{u, d}$ are real scalars. The VEV $v_{h}$ represents the Wilson line degree of freedom: $\alpha=\sqrt{2} g_{c} L v_{h}$. Thus, the value of $v_{h}$ is dynamically determined through the effective potential (3.38), as seen below. With a non-trivial value of $v_{h}$, the residual $U(1)$ gauge symmetry is broken and the zero-mode of $A_{\mu}^{3}$ acquires the mass of $M_{W}^{2}=\left(2 g_{c} v_{h}\right)^{2}$. 
We now focus on the physical Higgs mass spectrum. At the tree-level, the four real scalars $r_{u, d}$ and $\pi_{u, d}$ are rearranged as the mass eigenstates:

$$
\begin{aligned}
h & =\frac{1}{\sqrt{2}}\left(r_{u}+r_{d}\right), & H & =\frac{1}{\sqrt{2}}\left(-r_{u}+r_{d}\right), \\
A & =\frac{1}{\sqrt{2}}\left(\pi_{u}+\pi_{d}\right), & G & =\frac{1}{\sqrt{2}}\left(-\pi_{u}+\pi_{d}\right),
\end{aligned}
$$

where $h$ and $H$ are the CP-even Higgs scalars, $A$ is the CP-odd Higgs and $G$ is the mode eaten by the massive gauge boson. In terms of $5 \mathrm{D}$ language, $h, H, A$ and $G$ correspond to the zero-modes of $a_{2}, \sigma_{1}, \sigma_{2}$ and $a_{1}$, respectively. Using (3.39) in $V_{h}^{0}$, we observe that only $H$ have the non-zero mass as $m_{H}^{2}=M_{W}^{2}$ and the others are massless at the tree-level.

Since the one-loop potential lifts up both the flat and non-flat directions, all the Higgs masses are corrected. As long as the Higgs VEV lies along the flat direction, even at the oneloop level, mass eigenstates of the Higgs fields are same as (3.40). Putting the expansion (3.39) into $V_{h}^{1}$, the one-loop mass corrections are defined by

$$
\left.\delta m_{\phi}^{2} \equiv \frac{\partial^{2} V_{h}^{1}}{\partial \phi^{2}}\right|_{r, \pi \rightarrow 0},
$$

where $\phi=\{h, H, A, G\}$. The mass corrections to the physical Higgs modes are found to be

$$
\begin{aligned}
\delta m_{h}^{2}=\frac{3 g_{c}^{2} M_{W}^{2}}{2 \pi^{2} \alpha^{2}}\left[\left(2-N_{+}\right) \mathcal{C}_{\beta}^{+}-\right. & N_{-} \mathcal{C}_{\beta}^{-}+\frac{\alpha^{2}}{96}\left\{9 N_{+}-72+3 N_{-} \ln \left[\cos ^{2}(\pi \beta)\right]\right. \\
& \left.\left.+24 \ln \left[\frac{\alpha^{2}}{4 \sin ^{2}(\pi \beta)}\right]-3 N_{+} \ln \left[\frac{\alpha^{2}}{16 \sin ^{2}(\pi \beta)}\right]\right\}\right], \\
\delta m_{H}^{2}=\frac{g_{c}^{2} M_{W}^{2}}{2 \pi^{2} \alpha^{2}}\left[\left(2-N_{+}\right) \mathcal{C}_{\beta}^{+}-\right. & N_{-} \mathcal{C}_{\beta}^{-}+\frac{\alpha^{2}}{96}\left\{9 N_{+}-48+3 N_{-} \ln \left[\cos ^{2}(\pi \beta)\right]\right. \\
& \left.\left.+24 \ln \left[\frac{\alpha^{2}}{4 \sin ^{2}(\pi \beta)}\right]-3 N_{+} \ln \left[\frac{\alpha^{2}}{16 \sin ^{2}(\pi \beta)}\right]\right\}\right], \\
\delta m_{A}^{2}=\frac{g_{c}^{2} M_{W}^{2}\left[\left(2-N_{+}\right) \mathcal{C}_{\beta}^{+}-\right.}{2 \pi^{2} \alpha^{2}}\left[N_{-} \mathcal{C}_{\beta}^{-}+\frac{\alpha^{2}}{96}\left\{9 N_{+}-72+3 N_{-} \ln \left[\cos ^{2}(\pi \beta)\right]\right.\right. & \left.\left.+24 \ln \left[\frac{\alpha^{2}}{4 \sin ^{2}(\pi \beta)}\right]-3 N_{+} \ln \left[\frac{\alpha^{2}}{16 \sin ^{2}(\pi \beta)}\right]\right\}\right],
\end{aligned}
$$

where we use the notation $\alpha=\sqrt{2} g_{c} L v_{h}$. A simple relation $\delta m_{A}^{2}=\delta m_{h}^{2} / 3$ is observed. In the present case, the CP-odd Higgs $A$ always becomes lighter than the SB Higgs $h$; this may be affected by the introduction of matter multiplets in larger representations.

It should also be mentioned that the terms proportional to $\mathcal{C}_{\beta}^{+}$and $\mathcal{C}_{\beta}^{-}$appear as the particular combination, $\left(2-N_{+}\right) \mathcal{C}_{\beta}^{+}-N_{-} \mathcal{C}_{\beta}^{-}$, in all the mass corrections (3.41) , up to the overall 
factors. These contributions come from $\mathcal{O}\left(\left(g_{c} h_{u, d}\right)^{2}\right)$ part of the effective potential (3.38). As argued below, in order to obtain realistic vacua $(\alpha \ll 1)$, coefficient of the quadratic part of the potential should be suppressed. This requires $\left(2-N_{+}\right) \mathcal{C}_{\beta}^{+}-N_{-} \mathcal{C}_{\beta}^{-} \ll 1$ in the SB Higgs mass $\delta m_{h}^{2}$. Such the cancellation occurs simultaneously in $\delta m_{A}^{2}$ and $\delta m_{H}^{2}$ in the present analyses. The situation however may not be generally realized; if one adds matter multiplets in larger representations or considers models with larger gauge groups, then the cancellation may no longer occur in mass corrections of some of the non-SB Higgs scalars. In such cases, some of the non-SB Higgs masses could be enhanced compared to the SB Higgs mass through corrections from $\mathcal{O}\left(\left(g_{c} h_{u, d}\right)^{2}\right)$ part of the potential.

One can read the stationary condition of the potential from $\delta m_{G}^{2}=0$, which yields

$$
\begin{aligned}
3\left(2-N_{+}\right) \mathcal{C}_{\beta}^{+}-3 N_{-} \mathcal{C}_{\beta}^{-} & +\frac{\alpha^{2}}{96}\left\{11 N_{+}-88+3 N_{-} \ln \left[\cos ^{2}(\pi \beta)\right]\right. \\
+ & \left.24 \ln \left[\frac{\alpha^{2}}{4 \sin ^{2}(\pi \beta)}\right]-3 N_{+} \ln \left[\frac{\alpha^{2}}{16 \sin ^{2}(\pi \beta)}\right]\right\}=0
\end{aligned}
$$

for $\alpha \neq 0$. Using (3.42), one can eliminate the logarithmic terms in (3.41) and simplify the expressions as

$$
\begin{aligned}
\delta m_{h 0}^{2} & =\frac{3 g_{c}^{2} M_{W}^{2}}{2 \pi^{2} \alpha^{2}}\left[2\left(N_{+}-2\right) \mathcal{C}_{\beta}^{+}+2 N_{-} \mathcal{C}_{\beta}^{-}+\frac{\alpha^{2}}{96}\left[16-2 N_{+}\right]\right] \\
\delta m_{H 0}^{2} & =\frac{g_{c}^{2} M_{W}^{2}}{2 \pi^{2} \alpha^{2}}\left[2\left(N_{+}-2\right) \mathcal{C}_{\beta}^{+}+2 N_{-} \mathcal{C}_{\beta}^{-}+\frac{\alpha^{2}}{96}\left[40-2 N_{+}\right]\right]
\end{aligned}
$$

These expressions are only valid at the minimum of the one-loop effective potential (3.38); it is implied by the subscripts 0 in the left hand side in (3.43). From the expressions of (3.43), it is expected that the ratio between the mass corrections to the SB Higgs $h$ and non-SB Higgs $H$ takes order one values. As argued previously, to obtain a phenomenologically viable Higgs mass spectrum, the one-loop mass correction to the SB Higgs should not be suppressed more than the weak scale. Thus, we expect that the correction to the tree-massive Higgs $H$ is not negligible compared to the tree-level mass, as discussed in Section 2 ,

Let us study the mass corrections to Higgs scalars with the dynamically determined VEV of the flat direction $\alpha$. The flat limit potential is obtained by the substitution $h_{u}=h_{d} \rightarrow$ $v_{h} / \sqrt{2}=\alpha /\left(2 g_{c} L\right)$ in (3.38):

$$
\left.V_{\text {flat }}(\alpha) \equiv V_{h}^{1}\left(h_{u}, h_{d}\right)\right|_{h_{u}=h_{d} \rightarrow \alpha /\left(2 g_{c} L\right)} .
$$

If one fix the parameters $\beta, N_{+}$and $N_{-}$, then the value of $\alpha$ at the minimum of the flat limit potential is dynamically determined; we denote the value as $\alpha_{0}$. A non-trivial value of $\alpha_{0}$ leads 

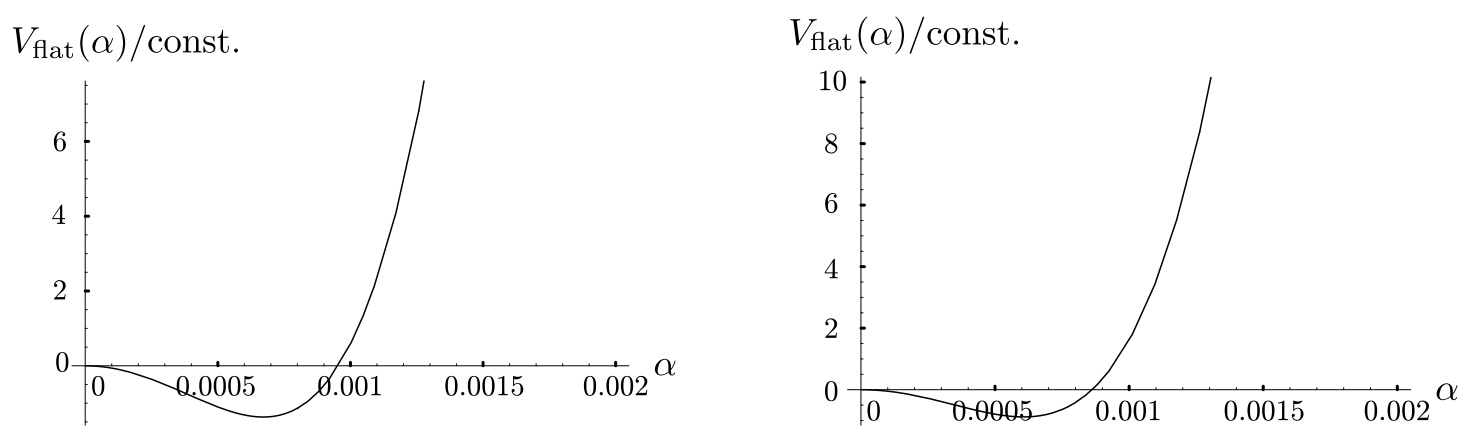

Figure 2: The one-loop Higgs potential along the flat direction $V_{\text {flat }}$ as the function of $\alpha$, where we take particular normalization of the potential. The left figure shows the case with $\left(N_{+}, N_{-}\right)=(10,15)$ and $\beta \simeq 0.211$ and the right one shows the case with $\left(N_{+}, N_{-}\right)=(30,45)$ and $\beta \simeq 0.263$. The potential is minimized with $\alpha_{0}=6.71 \times 10^{-4}$ and $\alpha=6.01 \times 10^{-4}$ for the left and right cases, respectively.

to spontaneous breaking of the residual $U(1)$ gauge symmetry. Since the approximation used to derive (3.38) is only valid for $\alpha_{0} \ll \beta \lesssim 1$, we consider such the minima in the analyses.

It is known that a suppressed value of $\alpha_{0}$ is obtained when the coefficient of the $\alpha^{2}$ term takes a small negative value and of the $\alpha^{4}$ term is a positive value in the flat limit potential [11, 17]. From (3.32), one can see that the contributions to the quadratic term from the vector multiplet is positive. In addition, from (3.36) and fig. 1, the contribution from $\eta_{U}=+1\left(\eta_{U}=-1\right)$ hypermultiplets is realized as negative (positive). Thus, $\eta_{U}=+1$ hypermultiplets play the role to decrease the coefficient; for $N_{+} \geq 3$, the coefficient can be negative. Moreover, using the positive contributions from $\eta_{U}=-1$ hypermultiplets, one can obtain a small negative coefficient in the present case.

In order to increase the one-loop mass correction to the SB Higgs, a large positive coefficient of the $\alpha^{4}$ term in (3.41) is preferred. The correction to the coefficient from the vector multiplet is negative and from $\eta_{U}=+1\left(\eta_{U}=-1\right)$ hypermultiplets is positive (negative small). To obtain a positive value, one must introduce relatively large number of $\eta_{U}=+1$ hypermultiplets since contribution to the $\alpha^{4}$ term from a hypermultiplet in the fundamental representation is suppressed by the factor of $1 / 2^{4}$ compared to one from the vector multiplet. For the case with $N_{+} \geq 9$, the coefficient takes a positive value and can be enhanced by the logarithmic factors in (3.41) for $\alpha_{0} \ll \beta$ [11].

As the explicit examples, we show the cases with $\left(N_{+}, N_{-}\right)=(10,15)$ and $\left(N_{+}, N_{-}\right)=$ $(30,45)$. The number of bulk fields seems to be rather large; note that if one introduces 

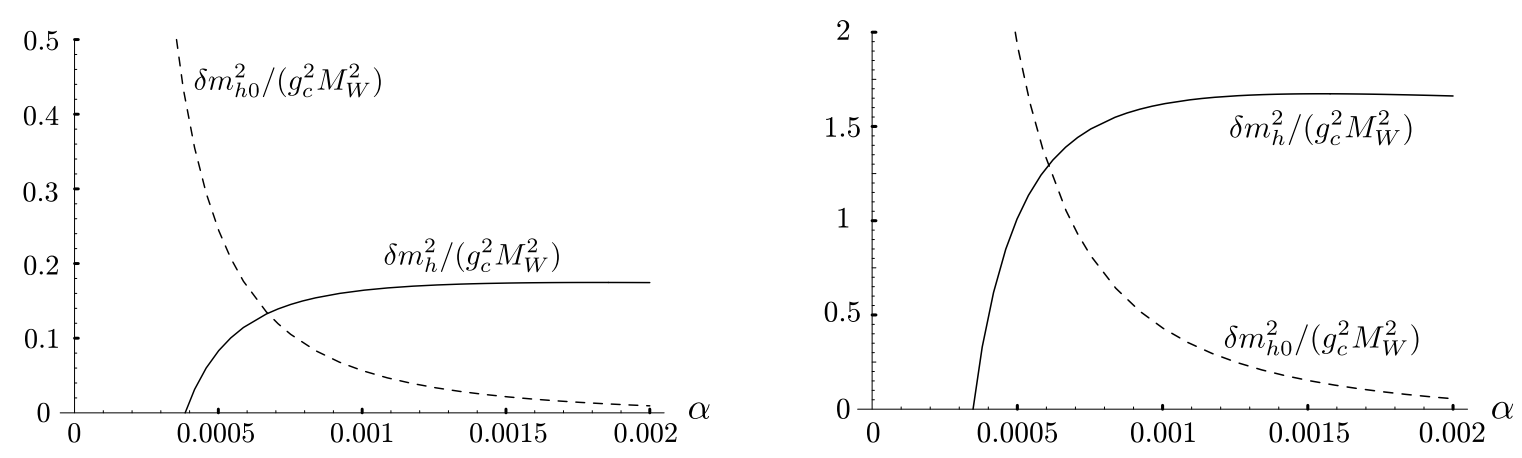

Figure 3: The values of $\delta m_{h}^{2} /\left(g_{c}^{2} M_{W}^{2}\right)$ (solid) and $\delta m_{h 0}^{2} /\left(g_{c}^{2} M_{W}^{2}\right)$ (dashed) as the functions of $\alpha$. The left figure shows the case with $\left(N_{+}, N_{-}\right)=(10,15)$ and $\beta \simeq 0.211$ and the right one shows the case with $\left(N_{+}, N_{-}\right)=(30,45)$ and $\beta \simeq 0.263$. For the illustrative purpose, we draw both the solid and dashed lines in each figure: at the minima of the potential, solid and dashed lines indicate the same values. We obtain $\delta m_{h}^{2} /\left(g_{c}^{2} M_{W}^{2}\right)=0.133$ and $\delta m_{h}^{2} /\left(g_{c}^{2} M_{W}^{2}\right)=1.32$ for the left and right cases, respectively.

hypermultiplets in the adjoint (or larger) representation, then a few pieces of bulk fields are sufficient to obtain enough heavy Higgs fields [11, 17]. While we don't address the issues in the present analyses, introduction of the large representations is straightforward. The typical behavior of the potential is shown as the function of $\alpha$ in fig. 2, where we take particular normalization for the overall scale of the potential. From the figures, one can see that $\alpha_{0} \ll \beta \lesssim 1$ is actually realized; hence, the approximation used to derive the effective potential is valid around the minima. The numerical evaluation indicates that $\alpha_{0}=6.71 \times 10^{-4}$ and $\alpha_{0}=6.01 \times 10^{-4}$ for the cases with $\left(N_{+}, N_{-}\right)=(10,15)$ and $\left(N_{+}, N_{-}\right)=(30,45)$, respectively. The residual $U(1)$ gauge symmetry is broken for both cases.

In fig. 3, the mass corrections to SB Higgs field $h$ are shown as the function of $\alpha$. The solid and dashed lines correspond to $\delta m_{h}^{2} /\left(g_{c}^{2} M_{W}^{2}\right)$ and $\delta m_{h 0}^{2} /\left(g_{c}^{2} M_{W}^{2}\right)$, respectively. For the illustrative purpose, we draw both the lines in each figure: at the minima of the potential, both the lines indicate same values. We obtain $\delta m_{h}^{2} /\left(g_{c}^{2} M_{W}^{2}\right)=0.133$ and $\delta m_{h}^{2} /\left(g_{c}^{2} M_{W}^{2}\right)=1.32$ for the cases with $\left(N_{+}, N_{-}\right)=(10,15)$ and $\left(N_{+}, N_{-}\right)=(30,45)$ at the minima, respectively. As mentioned before, the values of the corrections are straightforwardly increased by incorporating more bulk fields and/or large representations [10, 11, 17].

Finally, we show the mass correction to non-SB Higgs $H$. We observe that $\alpha$-dependence of the mass correction to $H$ is not so different from the case of the SB Higgs $h$. For comparison between the mass corrections to $h$ and $H$, we present the ratio between the corrections in 

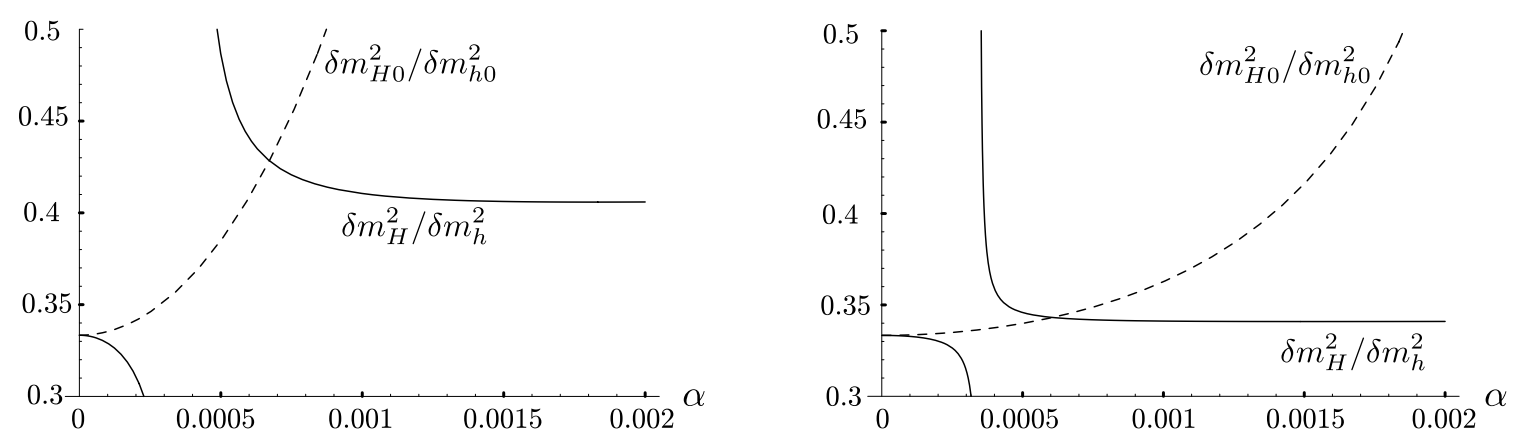

Figure 4: The values of $\delta m_{H}^{2} / \delta m_{h}^{2}$ (solid) and $\delta m_{H 0}^{2} / \delta m_{h 0}^{2}$ (dashed) as the functions of $\alpha$. The left figure shows the case with $\left(N_{+}, N_{-}\right)=(10,15)$ and $\beta \simeq 0.211$ and the right one shows the case with $\left(N_{+}, N_{-}\right)=(30,45)$ and $\beta \simeq 0.263$. As in fig. 3, solid and dashed lines indicate same values at the minima of the potential; we obtain $\delta m_{H}^{2} / \delta m_{h}^{2}=0.429$ and for $\delta m_{H}^{2} / \delta m_{h}^{2}=0.343$ the left and right cases, respectively.

fig. 4: the solid and dashed lines correspond to $\delta m_{H}^{2} / \delta m_{h}^{2}$ and $\delta m_{H 0}^{2} / \delta m_{h 0}^{2}$, respectively. At the point where $\delta m_{h}^{2}$ goes to zero, $\delta m_{H}^{2} / \delta m_{h}^{2}$ is strongly enhanced. For a small value of $\alpha$, $\delta m_{h}^{2}$ and/or $\delta m_{H}^{2}$ become negative; thus, the solid lines in each figure are disconnected as the functions of $\alpha$. The solid and dashed lines are crossed at the minima of the potential; we find $\delta m_{H}^{2} / \delta m_{h}^{2}=0.429$ and $\delta m_{H}^{2} / \delta m_{h}^{2}=0.343$ for the cases with $\left(N_{+}, N_{-}\right)=(10,15)$ and $\left(N_{+}, N_{-}\right)=(30,45)$ at the minima, respectively. There is no tachyonic mode in the physical Higgs spectrum; hence, local stability of the minima is confirmed. The one-loop mass correction to the tree-massive mode is not so suppressed rather than the tree-level value. The loop correction thus brings important effects not only on the tree-massless Higgs scalars, but also on the tree-massive Higgs scalar, as expected.

\section{Summary and perspective}

In this paper, we considered the 5D SU(2) SUSY model. The model leads to the vector-like pair of Higgs scalars at low energy. We explicitly analyzed the one-loop corrected multi-Higgs mass spectrum based on the useful approximation scheme for the effective potential including all the Higgs backgrounds. We focused on the mass correction for the non-SB (tree-massive) Higgs and found that both the tree-massless and massive Higgs scalars obtain the finite mass collections of similar size from the loop effects.

The results in the analyses implies that the one-loop mass corrections are important not only for the tree-massless modes but also for the massive modes in several realistic models. For 
example, in the 5D $S U(3)_{c} \times S U(3)_{W}$ SUSY model [10], two-Higgs doublets appear at a lowenergy regime. After the electroweak symmetry breaking, physical Higgs spectrum consists of two CP-even Higgs scalars $h$ and $H$, a CP-odd Higgs scalar $A$ and a charged Higgs scalar $H^{ \pm}$. At the tree-level, some of them become massive; the one-loop corrections to their masses are expected to bring important effects. It is known that the two Higgs doublets are also predicted in the minimal supersymmetric standard model [31]. Except for soft SUSY breaking contributions, both models have similar structure of tree-level Higgs potential, namely the supersymmetric D-term potential. Since the quantum corrections are expected to play the important role to raise Higgs masses in both models, comparison between the Higgs spectra is interesting. In 6D setup, it is also known to appear two Higgs doublets, and the similar Higgs contents to the above models are expected. We expect again that the one-loop effects modify the multi-Higgs mass spectrum. It is important to estimate the quantum corrections for the Higgs masses in view of high-energy experiments.

Moreover, it is an interesting subject to give detailed study of vacuum structure in the above models. In general, minima deviated from the flat directions of tree-level potential may be generated through the radiative corrections, while such minima are not found in our analyses. At the flat direction of the tree-level potential, the ratio between VEVs of the two Higgs doublets, so-called $\tan \beta$, is equal to one. However, if vacua are shifted to non-flat directions through radiative corrections, then $\tan \beta \neq 1$ is realized in the models. The Higgs mass spectra may be significantly changed by the shift of the vacua.

In addition, the perturbative calculation of the effective potential developed in this paper is useful for examining the $6 \mathrm{D}$ case; approximate behavior of the effective potential for a small VEV of the SB Higgs, which is not clear even in the flat limit calculation, can be revealed with the perturbative expansion of the potential. The examination of the above subjects are left to our future studies [32].

\section{Acknowledgments}

The authors would like to thank Professor Y. Hosotani (Osaka Univ.) for valuable discussions. K.K. would also thank to K. Harada (Kyushu Univ.) and A. Watanabe (Kyushu Univ.) for useful discussions. K.T. is partially supported by a Grant-in-Aid for Scientific Research (No. 18540275) from the Japanese Ministry of Education, Science, Sports and Culture and

also by the 21st Century COE Program at Tohoku University. T.Y. is supported in part by The 21st Century COE Program "Towards a New Basic Science; Depth and Synthesis". 


\section{A Background dependent operators}

Here we summarize the operators appearing in the functional determinants (3.27) and (3.34). Note that in the determinants, using unitary transformations, one can freely change the basis of the operators in the representation space. Thus, the following sets of the forms are realized in a particular basis. For the contributions from vector multiplet in (3.27), the operators are written as follows:

$$
\begin{aligned}
& \Delta_{0}\left(A_{\mu}^{(0)}\right)=\square, \quad \Delta_{0}\left(Q^{(0)}\right)=\square \cdot \mathbf{1}_{2 \times 2}, \\
& \Delta_{0}\left(A_{\mu}^{(n)}\right)=\Delta_{0}\left(Q^{(n)}\right)=\left[\square+(n / R)^{2}\right] \cdot \mathbf{1}_{3 \times 3}, \quad m_{A_{\mu}}^{(n)}=m_{Q}^{(n)}=(n / R), \\
& \Delta_{0}\left(\lambda^{(n)}\right)=\left[\square+\left(\frac{\beta+n}{R}\right)^{2}\right] \cdot \mathbf{1}_{3 \times 3}, \quad m_{\lambda}^{(n)}=(\beta+n) / R, \\
& \Delta_{1}\left(A_{\mu}^{(n)}\right)=\Delta_{1}\left(Q^{(n)}\right)=\Delta_{1}\left(\lambda^{(n)}\right)=\sqrt{2} g\left(\begin{array}{ccc}
0 & 0 & n_{u}+n_{d}^{*} \\
0 & 0 & n_{u}^{*}+n_{d} \\
n_{u}^{*}+n_{d} & n_{u}+n_{d}^{*} & 0
\end{array}\right) \\
& \Delta_{2}\left(A_{\mu}^{(0)}\right)=2 g^{2}\left(\left|n_{u}\right|^{2}+\left|n_{d}\right|^{2}\right) \\
& \Delta_{2}\left(A_{\mu}^{(n)}\right)=g^{2}\left(\begin{array}{ccc}
\left|n_{u}\right|^{2}+\left|n_{d}\right|^{2} & 2 n_{u} n_{d}^{*} & 0 \\
2 n_{d} n_{u}^{*} & \left|n_{u}\right|^{2}+\left|n_{d}\right|^{2} & 0 \\
0 & 0 & 2\left(\left|n_{u}\right|^{2}+\left|n_{d}\right|^{2}\right)
\end{array}\right) \text {, } \\
& \Delta_{2}\left(Q^{(0)}\right)=g^{2}\left(\begin{array}{cc}
3\left|n_{u}\right|^{2}-\left|n_{d}\right|^{2} & 2 n_{u} n_{d}^{*} \\
2 n_{d} n_{u}^{*} & 3\left|n_{d}\right|^{2}-\left|n_{u}\right|^{2}
\end{array}\right) \\
& \Delta_{2}\left(Q^{(n)}\right)=g^{2}\left(\begin{array}{ccc}
3\left|n_{u}\right|^{2}-\left|n_{d}\right|^{2} & 2 n_{u} n_{d}^{*} & 0 \\
2 n_{d} n_{u}^{*} & 3\left|n_{d}\right|^{2}-\left|n_{u}\right|^{2} & 0 \\
0 & 0 & 2\left(\left|n_{u}\right|^{2}+\left|n_{d}\right|^{2}\right)
\end{array}\right) \text {, } \\
& \Delta_{2}\left(\lambda^{(n)}\right)=2 g^{2}\left(\begin{array}{ccc}
\left|n_{d}\right|^{2} & n_{u} n_{d}^{*} & 0 \\
n_{u}^{*} n_{d} & \left|n_{u}\right|^{2} & 0 \\
0 & 0 & \left|n_{u}\right|^{2}+\left|n_{d}\right|^{2}
\end{array}\right)
\end{aligned}
$$

For the contributions from hypermultiplets in (3.34), the operators are written as follows:

$$
\begin{aligned}
& \Delta_{0}\left(\phi_{+}^{(n)}\right)=\left[\square+\left(\frac{\beta+n}{R}\right)^{2}\right] \cdot \mathbf{1}_{2 \times 2}, \quad m_{\phi_{+}}^{(n)}=(\beta+n) / R, \\
& \Delta_{0}\left(\phi_{-}^{(n)}\right)=\left[\square+\left(\frac{\beta+n-1 / 2}{R}\right)^{2}\right] \cdot \mathbf{1}_{2 \times 2}, \quad m_{\phi_{-}}^{(n)}=(\beta+n-1 / 2) / R, \\
& \Delta_{0}\left(\psi^{(0)}\right)=\square, \\
& \Delta_{0}\left(\psi_{+}^{(n)}\right)=\left[\square+(n / R)^{2}\right] \cdot \mathbf{1}_{2 \times 2}, \quad m_{\psi_{+}}^{(n)}=n / R, \\
& \Delta_{0}\left(\psi_{-}^{(n)}\right)=\left[\square+\left(\frac{n-1 / 2}{R}\right)^{2}\right] \cdot \mathbf{1}_{2 \times 2}, \quad m_{\psi_{-}}^{(n)}=(n-1 / 2) / R,
\end{aligned}
$$




$$
\begin{aligned}
\Delta_{1}\left(\phi^{(n)}\right) & =\Delta_{1}\left(\psi^{(n)}\right)=g\left(\begin{array}{cc}
0 & n_{u}+n_{d}^{*} \\
n_{u}^{*}+n_{d} & 0
\end{array}\right), \\
\Delta_{2}\left(\phi^{(n)}\right) & =\frac{g^{2}}{2}\left(\left|n_{u}\right|^{2}+\left|n_{d}\right|^{2}\right) \cdot \mathbf{1}_{2 \times 2}, \\
\Delta_{2}\left(\psi_{u, d}^{(0)}\right) & =g^{2}\left|n_{u, d}\right|^{2}, \\
\Delta_{2}\left(\psi^{(n)}\right) & =g^{2}\left(\begin{array}{cc}
\left|n_{u}\right|^{2} & 0 \\
0 & \left|n_{d}\right|^{2}
\end{array}\right) .
\end{aligned}
$$

\section{B Loop integrals with K-K mode summation}

When one evaluates the functional determinant with the perturbative expansion (3.21), loop integrals with K-K mode summation appear. Here the evaluations of the integrals and summations are summarized.

At first, we introduce the functions, which include 4D loop integral and K-K mode summation, as

$$
\hat{\zeta}_{[x, m, \gamma]}=\lim _{\mu \rightarrow 0} \sum_{n=-\infty}^{\infty} \int \frac{d^{4} p_{E}}{(2 \pi)^{4}} \frac{\left(\frac{n+\gamma}{R}\right)^{2 m}}{\left[p_{E}^{2}+\left(\frac{n+\gamma}{R}\right)^{2}+(\mu / R)^{2}\right]^{x}}
$$

where $p_{E}$ is Wick-rotated momentum. A dimensionless parameter $\mu$ is introduced in the propagator to regulate IR divergences if any, and we take a limit $\mu \rightarrow 0$ after the integration.

For the calculation of the determinant up to $\mathcal{O}\left(\left(g n_{u, d}\right)^{4}\right)$, one needs $\hat{\zeta}_{[1,0, \gamma]}, \hat{\zeta}_{[2,1, \gamma]}, \hat{\zeta}_{[2,0, \gamma]}$, $\hat{\zeta}_{[3,1, \gamma]}$ and $\hat{\zeta}_{[4,2, \gamma]}$; we consider only them here. Among them, $\hat{\zeta}_{[1,0, \gamma]}$ and $\hat{\zeta}_{[2,1, \gamma]}$ suffers UV divergences from the loop integral, and $\hat{\zeta}_{[3,1, \gamma]}$ and $\hat{\zeta}_{[4,2, \gamma]}$ suffer IR divergences only. Both the divergences are involved in $\hat{\zeta}_{[2,0, \gamma]}$. In addition, infinite summation of the K-K modes also bring the divergence. As shown below, the singularity arising from the summation is realized as UV divergence in view of 5D theory. Thus, one should evaluate all the functions with a suitable regularization both of the UV and IR singularities, even if 4D loop integral does not have UV divergence. Using the dimensional regularization, we evaluate UV divergences as

$$
\begin{aligned}
\int \frac{d^{4} p_{E}}{(2 \pi)^{4}} \frac{1}{\left[p_{E}^{2}+\Delta\right]^{x}} & \rightarrow M_{R G}^{4-d} \int \frac{d^{d} p_{E}}{(2 \pi)^{d}} \frac{1}{\left[p_{E}^{2}+\Delta\right]^{x}} \\
& =\frac{M_{R G}^{4-d}}{(4 \pi)^{d / 2} \Gamma(x)} \int_{0}^{\infty} d t t^{(x-d / 2)-1} e^{-\Delta t}
\end{aligned}
$$

where $d \rightarrow 4$ is implied in the expression. We introduce the renormalization scale $M_{R G}$ in order to keep the integral having mass dimension $4-2 x$. 
To carry out the summation in (B.1), we use the Poisson resummation formulae:

$$
\sum_{n=-\infty}^{\infty} f((n+\gamma))=\sum_{w=-\infty}^{\infty} e^{2 \pi i w \gamma} \int_{-\infty}^{\infty} d p f(p) e^{2 \pi i p w}
$$

Then, in the summation over $w$, only $w=0$ term has local divergence and $w \neq 0$ terms are regarded as non-local effects, namely UV finite. Hence, we divide $\hat{\zeta}_{[x, m, \gamma]}$ into two parts as

$$
\hat{\zeta}_{[x, m, \gamma]}=\hat{\zeta}_{[x, m, \gamma]}^{(w=0)}+\hat{\zeta}_{[x, m, \gamma]}^{(w \neq 0)}
$$

where $\hat{\zeta}_{[x, m, \gamma]}^{(w=0)}$ is the $w=0$ term in the summation and $\hat{\zeta}_{[x, m, \gamma]}^{(w \neq 0)}$ is sum of the other terms. The locally divergent terms are listed as follows:

$$
\hat{\zeta}_{[1,0, \gamma]}^{(w=0)}=\mathcal{I}_{1}, \quad \hat{\zeta}_{[2,1, \gamma]}^{(w=0)}=\frac{\mathcal{I}_{1}}{2}, \quad \hat{\zeta}_{[2,0, \gamma]}^{(w=0)}=\mathcal{I}_{2}, \quad \hat{\zeta}_{[3,1, \gamma]}^{(w=0)}=\frac{\mathcal{I}_{2}}{4}, \quad \hat{\zeta}_{[4,2, \gamma]}^{(w=0)}=\frac{\mathcal{I}_{2}}{8},
$$

where

$$
\mathcal{I}_{m}=2 \pi R M_{R G}^{5-(d+1)} \lim _{\mu \rightarrow 0} \int \frac{d^{d+1} p_{E}}{(2 \pi)^{d+1}} \frac{1}{\left[p_{E}^{2}+(\mu / R)^{2}\right]^{m}} .
$$

One can observe that the divergences arising from 4D loop integral and K-K mode summation are written by the 5D Lorentz invariant forms. The other non-local UV finite effects are involved in $w \neq 0$ terms, and violate 5D Lorentz invariance.

While $w \neq 0$ terms are UV finite, some of them involve IR divergences, as argued. For $\hat{\zeta}_{[1,0, \gamma]}^{(w \neq 0)}$ and $\hat{\zeta}_{[2,1, \gamma]}^{(w \neq 0)}$, there is no worse IR behavior and the integrals can be naively evaluated with $\mu=0$. Then we observe

$$
\hat{\zeta}_{[1,0, \gamma]}^{(w \neq 0)}=-\hat{\zeta}_{[2,1, \gamma]}^{(w \neq 0)}=2 \frac{2 \pi R}{64 \pi^{5} R^{3}} \sum_{w=1}^{\infty} \frac{\cos (2 \pi w \gamma)}{w^{3}} .
$$

For the others, one should carefully evaluate the following integrals with non-zero $\mu$ and $\gamma$ :

$$
\begin{aligned}
\hat{\zeta}_{[2,0, \gamma]}^{(w \neq 0)=} & \lim _{\mu \rightarrow 0} \frac{2 \sqrt{\pi} R}{(4 \pi)^{2}} \sum_{w=1}^{\infty} \cos (2 \pi w \gamma) \int_{0}^{\infty} d t t^{-1 / 2-1} e^{-(\mu / R)^{2} t} e^{-(\pi w R)^{2} / t} \\
\hat{\zeta}_{[3,1, \gamma]}^{(w \neq 0)}= & \lim _{\mu \rightarrow 0} \frac{-\sqrt{\pi} R}{2 \cdot \Gamma(3) \cdot(4 \pi)^{2}} \sum_{w=1}^{\infty} \cos (2 \pi w \gamma) \\
& \times \int_{0}^{\infty} d t\left[(2 \pi w R)^{2} t^{-3 / 2-1}-2 t^{-1 / 2-1}\right] e^{-(\mu / R)^{2} t} e^{-(\pi w R)^{2} / t} \\
\hat{\zeta}_{[4,2, \gamma]}^{(w \neq 0)=} & \lim _{\mu \rightarrow 0} \frac{\sqrt{\pi} R}{8 \cdot \Gamma(4) \cdot(4 \pi)^{2}} \sum_{w=1}^{\infty} \cos (2 \pi w \gamma) \\
& \times \int_{0}^{\infty} d t\left[(2 \pi w R)^{4} t^{-5 / 2-1}-12(2 \pi w R)^{2} t^{-3 / 2-1}+12 t^{-1 / 2-1}\right] e^{-(\mu / R)^{2} t} e^{-(\pi w R)^{2} / t},
\end{aligned}
$$


where $d \rightarrow 4$ are taken since they have no UV divergences due to the exponential factors including $\omega \neq 0$. Here UV limit corresponds to $t \rightarrow 0$ in each $t$-integral. On the other hand, IR limit corresponds to $t \rightarrow \infty$ and is suitably regularized by non-zero $\mu$. After the loop integral, appropriate limit should be taken for $\mu$ and $\gamma$. The results are listed for the case with $\gamma=0$ and $\gamma \neq 0$ as

$$
\begin{array}{ll}
\hat{\zeta}_{[2,0, \gamma \neq 0]}^{(w \neq 0)}=-2 \frac{2 \pi R}{64 \pi^{3} R} \ln \left[4 \sin ^{2}(\pi \gamma)\right]+\mathcal{O}(\mu), & \hat{\zeta}_{[2,0,0]}^{(w \neq 0)}=-2 \frac{2 \pi R}{64 \pi^{3} R} \ln \left[(2 \pi \mu)^{2}\right]+\mathcal{O}(\mu), \\
\hat{\zeta}_{[3,1, \gamma \neq 0]}^{(w \neq 0)}=\mathcal{O}(\mu), & \hat{\zeta}_{[3,1,0]}^{(w \neq 0)}=-\frac{2 \pi R}{64 \pi^{3} R}+\mathcal{O}(\mu), \\
\hat{\zeta}_{[4,2, \gamma \neq 0]}^{(w \neq 0)}=\mathcal{O}(\mu), & \hat{\zeta}_{[4,2,0]}^{(w \neq 0)}=-\frac{1}{3} \frac{2 \pi R}{64 \pi^{3} R}+\mathcal{O}(\mu),
\end{array}
$$

where $\mu \rightarrow 0$ is understood. Note that $\hat{\zeta}_{[2,0,0]}^{(w \neq 0)}$ includes $\log (\mu)$, which increases with $\mu \rightarrow 0$. In the calculation of the effective potential, this $\mu$-dependence would be canceled out by the other IR divergence. In our example of the calculation in Section 3 , IR divergences are also involved in the resummation of the zero-mode contributions in (3.28). Both the IR divergences in $\hat{\zeta}_{[2,0,0]}^{(w \neq 0)}$ and in the resummation are canceled out in the one-loop contribution; hence, the effective potential does not depend on the artificial parameter $\mu$.

Finally we define the functions:

$$
\mathcal{F}_{[x, m, \beta]}=\frac{1}{2 \pi R}\left[\hat{\zeta}_{[x, m, 0]}-\hat{\zeta}_{[x, m, \beta]}\right],
$$

which appears when contributions from bosonic and fermionic fluctuations are summed in SUSY theories. In the functions, all the UV divergences are canceled out, and thus there are only the UV finite terms. For a finite value of $\beta \neq 0$, they are given as follows:

$$
\begin{aligned}
& \mathcal{F}_{[1,0, \beta]}=\frac{1}{64 \pi^{5} R^{3}} \sum_{w=1}^{\infty} \frac{4 \sin ^{2}(\pi w \beta)}{w^{3}}, \\
& \mathcal{F}_{[2,1, \beta]}=-\frac{1}{64 \pi^{5} R^{3}} \sum_{w=1}^{\infty} \frac{4 \sin ^{2}(\pi w \beta)}{w^{3}}, \\
& \mathcal{F}_{[2,0, \beta]}=\frac{2}{64 \pi^{3} R} \ln \left[\frac{4 \sin ^{2}(\pi \beta)}{(2 \pi \mu)^{2}}\right], \\
& \mathcal{F}_{[3,1, \beta]}=-\frac{1}{64 \pi^{3} R}, \\
& \mathcal{F}_{[4,2, \beta]}=-\frac{1}{3 \cdot 64 \pi^{3} R},
\end{aligned}
$$

where $\mu \rightarrow 0$ is understood. For evaluation of the contributions from $\eta_{U}=-1$ fields, it is useful to define the function as

$$
\mathcal{F}_{[x, m, \beta]}^{\prime}=\frac{1}{2 \pi R}\left[\hat{\zeta}_{[x, m,-1 / 2]}-\hat{\zeta}_{[x, m, \beta-1 / 2]}\right] .
$$


It can be estimated as

$$
\begin{aligned}
& \mathcal{F}_{[1,0, \beta]}^{\prime}=\frac{1}{64 \pi^{5} R^{3}} \sum_{w=1}^{\infty} \frac{4(-1)^{w} \sin ^{2}(\pi w \beta)}{w^{3}} \\
& \mathcal{F}_{[2,1, \beta]}^{\prime}=-\frac{1}{64 \pi^{5} R^{3}} \sum_{w=1}^{\infty} \frac{4(-1)^{w} \sin ^{2}(\pi w \beta)}{w^{3}} \\
& \mathcal{F}_{[2,0, \beta]}^{\prime}=\frac{2}{64 \pi^{3} R} \ln \left[\cos ^{2}(\pi \beta)\right]
\end{aligned}
$$

and $\mathcal{F}_{[3,1, \beta]}^{\prime}=\mathcal{F}_{[4,2, \beta]}^{\prime}=0$. 


\section{References}

[1] N. S. Manton, Nucl. Phys. B 158 (1979) 141.

[2] D. B. Fairlie, Phys. Lett. B 82 (1979) 97.

[3] Y. Hosotani, Phys. Lett. B 126 (1983) 309; Annals Phys. 190 (1989) 233.

[4] N. V. Krasnikov, Phys. Lett. B 273 (1991) 246; H. Hatanaka, T. Inami and C. S. Lim, Mod. Phys. Lett. A 13 (1998) 2601; G. R. Dvali, S. Randjbar-Daemi and R. Tabbash, Phys. Rev. D 65 (2002) 064021; N. Arkani-Hamed, A. G. Cohen and H. Georgi, Phys. Lett. B 513 (2001) 232; I. Antoniadis, K. Benakli and M. Quiros, New J. Phys. 3 (2001) 20.

[5] M. Kubo, C. S. Lim and H. Yamashita, Mod. Phys. Lett. A 17 (2002) 2249; L. J. Hall, Y. Nomura and D. R. Smith, Nucl. Phys. B 639 (2002) 307; K. Takenaga, Phys. Rev. D 64 (2001) 066001; Phys. Rev. D 66 (2002) 085009; C. Csaki, C. Grojean and H. Murayama, Phys. Rev. D 67, (2003) 085012; G. Burdman and Y. Nomura, Nucl. Phys. B 656 (2003) 3; N. Haba, M. Harada, Y. Hosotani and Y. Kawamura, Nucl. Phys. B 657, (2003) 169 [Erratum-ibid. B 669, (2003) 381]; C. A. Scrucca, M. Serone and L. Silvestrini, Nucl. Phys. B 669 (2003) 128; I. Gogoladze, Y. Mimura, S. Nandi and K. Tobe, Phys. Lett. B 575, (2003) 66; C. Csaki, C. Grojean, H. Murayama, L. Pilo and J. Terning, Phys. Rev. D 69 (2004) 055006.

[6] G. Panico, M. Serone and A. Wulzer, Nucl. Phys. B 739 (2006) 186; G. Panico and M. Serone, JHEP 0505 (2005) 024; N. Maru and K. Takenaga, Phys. Rev. D 72 (2005) 046003; M. Sakamoto and K. Takenaga, Phys. Rev. D 75 (2007) 045015; Phys. Rev. D 76 (2007) 085016; N. Haba, K. Takenaga and T. Yamashita, Phys. Lett. B 605 (2005) 355 .

[7] A. T. Davies and A. McLachlan, Phys. Lett. B 200 (1988) 305; Nucl. Phys. B 317 (1989) 237; J. E. Hetrick and C. L. Ho, Phys. Rev. D 40 (1989) 4085; A. Higuchi and L. Parker, Phys. Rev. D 37 (1988) 2853; C. L. Ho and Y. Hosotani, Nucl. Phys. B 345 (1990) 445; A. McLachlan, Nucl. Phys. B 338 (1990) 188; K. Takenaga, Phys. Lett. B 425 (1998) 114 .

[8] R. Contino, Y. Nomura and A. Pomarol, Nucl. Phys. B 671 (2003) 148; K. y. Oda and A. Weiler, Phys. Lett. B 606 (2005) 408; K. Agashe, R. Contino and A. Pomarol, 
Nucl. Phys. B 719 (2005) 165; Y. Hosotani and M. Mabe, Phys. Lett. B 615 (2005) 257; Y. Hosotani, S. Noda, Y. Sakamura and S. Shimasaki, Phys. Rev. D 73 (2006) 096006; Y. Sakamura and Y. Hosotani, Phys. Lett. B 645 (2007) 442; Y. Hosotani and Y. Sakamura, arXiv:hep-ph/0703212 Y. Sakamura, Phys. Rev. D 76 (2007) 065002.

[9] Y. Hosotani, S. Noda and K. Takenaga, Phys. Rev. D 69 (2004) 125014; Phys. Lett. B $607(2005) 276$.

[10] N. Haba, Y. Hosotani, Y. Kawamura and T. Yamashita, Phys. Rev. D 70 (2004) 015010; N. Haba and T. Yamashita, JHEP 0404 (2004) 016.

[11] N. Haba, K. Takenaga and T. Yamashita, Phys. Lett. B 615 (2005) 247.

[12] N. Maru and K. Takenaga, Phys. Lett. B 637 (2006) 287.

[13] N. Maru and T. Yamashita, Nucl. Phys. B 754, 127 (2006); Y. Hosotani, N. Maru, K. Takenaga and T. Yamashita, Prog. Theor. Phys. 1181053 (2007).

[14] N. Haba, K. Takenaga and T. Yamashita, Phys. Rev. D 71 (2005) 025006.

[15] N. Arkani-Hamed, T. Gregoire and J. G. Wacker, JHEP 0203 (2002) 055.

[16] W.M. Yao et al. [Particle Data Group], J. Phys. G33 (2006) 1.

[17] G. Cacciapaglia, C. Csaki and S. C. Park, JHEP 0603 (2006) 099.

[18] M. F. Sohnius, Phys. Rept. 128 (1985) 39; A. Pomarol and M. Quiros, Phys. Lett. B 438 (1998) 255.

[19] M. Quiros, arXiv:hep-ph/0302189.

[20] J. Scherk and J. H. Schwarz, Phys. Lett. B 82 (1979) 60; P. Fayet, Phys. Lett. B 159 (1985) 121; Nucl. Phys. B 263 (1986) 649.

[21] R. Barbieri, L. J. Hall and Y. Nomura, Nucl. Phys. B 624 (2002) 63.

[22] P. Fayet and J. Iliopoulos, Phys. Lett. B 51, (1974) 461.

[23] K. Takenaga, Phys. Rev. D 58 (1998) 026004.

[24] N. Haba and T. Yamashita, JHEP 0402 (2004) 059. 
[25] H. Georgi, A. K. Grant and G. Hailu, Phys. Lett. B 506 (2001) 207.

[26] S. G. Nibbelink and M. Hillenbach, Nucl. Phys. B 748 (2006) 60.

[27] S. R. Coleman and E. Weinberg, Phys. Rev. D 7 (1973) 1888.

[28] N. Arkani-Hamed, A. G. Cohen and H. Georgi, Phys. Lett. B 516 (2001) 395;

C. A. Scrucca, M. Serone, L. Silvestrini and F. Zwirner, Phys. Lett. B 525 (2002) 169.

[29] G. von Gersdorff, N. Irges and M. Quiros, Phys. Lett. B 551 (2003) 351; C. A. Scrucca, M. Serone, L. Silvestrini and A. Wulzer, JHEP 0402 (2004) 049; C. Biggio and M. Quiros, Nucl. Phys. B 703 (2004) 199.

[30] H. M. Lee, H. P. Nilles and M. Zucker, Nucl. Phys. B 680 (2004) 177.

[31] K. Inoue, A. Kakuto, H. Komatsu and S. Takeshita, Prog. Theor. Phys. 67 (1982) 1889.

[32] K. Kojima, K. Takenaga and T. Yamashita, Work in progress. 